\title{
Right Markov Processes and Systems of Semilinear Equations with Measure Data
}

\author{
Tomasz Klimsiak ${ }^{1}$
}

Received: 29 September 2014 / Accepted: 25 October 2015 / Published online: 5 November 2015

(C) The Author(s) 2015. This article is published with open access at Springerlink.com

\begin{abstract}
In the paper we prove the existence of probabilistic solutions to systems of the form $-A u=F(x, u)+\mu$, where $F$ satisfies a generalized sign condition and $\mu$ is a smooth measure. As for $A$ we assume that it is a generator of a Markov semigroup determined by a right Markov process whose resolvent is order compact on $L^{1}$. This class includes local and nonlocal operators corresponding to Dirichlet forms as well as some operators which are not in the variational form. To study the problem we introduce new concept of compactness property relating the underlying Markov process to almost everywhere convergence. We prove some useful properties of the compactness property and provide its characterization in terms of Meyer's property (L) of Markov processes and in terms of order compactness of the associated resolvent.
\end{abstract}

Keywords Right Markov processes · Dirichlet forms · Semilinear elliptic systems · Order compactness $\cdot$ Probabilistic potential theory $\cdot$ Measure data $\cdot$ Smooth measure

Mathematics Subject Classification (2010) 35A01 · 35D99 $\cdot$ 60J40 $\cdot 60 \mathrm{~J} 45$

\section{Introduction}

Let $E$ be a Radon metrizable topological space, $F: E \times \mathbb{R}^{N} \rightarrow \mathbb{R}^{N}, N \geq 1$, be a measurable function and let $\mu=\left(\mu_{1}, \ldots, \mu_{N}\right)$ be a smooth measure on $E$. In the present paper we investigate the problem of existence of solutions of the system

$$
-A u=F(x, u)+\mu \text {. }
$$

Tomasz Klimsiak

tomas@mat.uni.torun.pl

1 Faculty of Mathematics and Computer Science, Nicolaus Copernicus University, Chopina 12/18, 87-100 Toruń, Poland 
Here $A$ is the linear operator associated with a Markov semigroup $\left\{T_{t}, t \geq 0\right\}$ on $L^{1}(E ; m)$. Our only assumption on $\left\{T_{t}\right\}$ is that it is representable by some right Markov process $\mathbb{X}=\left(\left\{X_{t}, t \geq 0\right\},\left\{P_{x}, x \in E\right\}\right)$ on $E$, i.e. for every $t \geq 0$ and $f \in L^{1}(E ; m)$,

$$
\left(T_{t} f\right)(x)=E_{x} f\left(X_{t}\right) \equiv p_{t} f(x) \text { for } m \text {-a.e. } x \in E,
$$

where $E_{x}$ denotes the expectation with respect to the measure $P_{x}$. The class of operators associated with such semigroups is fairly wide. It includes important local and nonlocal operators corresponding to quasi-regular Dirichlet forms (see [23, 32, 34]) as well as interesting operators which are not in the variational form, like some classes of Ornstein-Uhlenbeck processes (see Example 5.7).

As for $F=\left(f_{1}, \ldots, f_{N}\right)$ we assume that it is continuous with respect to $u$ and satisfies the following sign condition:

$$
\langle F(x, y), y\rangle \leq G(x)|y|, \quad x \in E, y \in \mathbb{R}^{N}
$$

for some appropriately integrable positive function $G$ (see hypotheses (H1)-(H4) in Section 3).

The first problem we encounter when dealing with systems of the form (1.1) is to give suitable definition of a solution. The problem occurs even in the case of one linear equation with local operator of the form $A=\sum_{i, j=1}^{d} \frac{\partial}{\partial x_{j}}\left(a_{i j} \frac{\partial}{\partial x_{i}}\right)$, whose study goes back to the papers of Serrin [38] and Stampacchia [40]. Serrin [38] constructed an example of (discontinuous) coefficients $a_{i j}$ and nontrivial function $u$ having the property that $u \in W_{0}^{1, q}(D)$ for every $q<d /(d-1)$ and $u$ is the distributional solution of Eq. 1.1 with data $\mu=0, F=0$. Since it was known that in general one can not expect that a solution to Eq. 1.1 belongs to the space $W_{0}^{1, q}(D)$ with $q \geq d /(d-1)$, the problem of the definition of a solution to Eq. 1.1 ensuring uniqueness arose. Stampacchia [40] solved this problem by introducing the socalled definition by duality. Since his work the theory of scalar equations with measure data and local operators (linear and nonlinear of of Leray-Lions type) have attracted considerable attention (see $[4,12,13,16,18]$ for results for equations with smooth measures $\mu$; a nice account of the theory for equations with general measures has been given in [3]).

The case of nonlocal operators is much more involved. To our knowledge there were only few attempts to investigate scalar linear equation (1.1) with operator $A=\Delta^{\alpha}$ with $\alpha \in(0,1]$ by analytical methods (see $[1,26])$. To encompass broader class of operators and semilinear equations in [28] (see also [29]) a probabilistic definition of a solution of scalar problem (1.1) is proposed. The basic idea in [28] is to define a solution via a nonlinear Feynman-Kac formula. Namely, a solution of Eq. 1.1 is a measurable function $u: E \rightarrow \mathbb{R}$ such that

$$
u(x)=E_{x} \int_{0}^{\infty} F\left(X_{t}, u\left(X_{t}\right)\right) d t+E_{x} \int_{0}^{\infty} d A_{t}^{\mu}
$$

for $m$-a.e. $x \in E$, where $A^{\mu}$ is a continuous additive functional of the process $\mathbb{X}$ corresponding to the measure $\mu$ in the Revuz sense (see [19, 23, 32, 35]). In [28] it is proved that in case $N=1$ if $F$ is nonincreasing with respect to $u$ then under mild integrability assumptions on the data there exists a unique solution to Eq. 1.1. In fact, if $A$ is a uniformly divergence form operator then the probabilistic solution of Eq. 1.1 coincides with Stampacchia's solution by duality.

When studying systems (1.1) with $F$ satisfying merely sign condition (1.3) we encounter new difficulties, which roughly speaking pertain to weaker regularity of solution of Eq. 1.1 than in the scalar case and to "compactness properties". In [27] we have studied systems of the form (1.1) on bounded domain $D \subset \mathbb{R}^{d}$ with $A=\Delta$ subject to homogeneous Dirichlet 
boundary condition. In [27] it is observed that in general, if $F$ only satisfies the sign condition, one cannot expect that $F(\cdot, u) \in L^{1}(D ; m)$. Moreover, it may happen that the first integral on the right-hand side of Eq. 1.4 is infinite. This together with the comments given before show that for systems, even in the case of a uniformly elliptic divergence form operator, neither the distributional definition nor the probabilistic via the Feynman-Kac formula (1.4) are applicable. For these reasons in [27] more general than in [28, 29] probabilistic definition of a solution of Eq. 1.1 is adopted. It uses the representation of $u$ in terms of some backward stochastic differential equation (BSDE) associated with $A, F, \mu$ (in case $F(\cdot, u$ ) is integrable the representation reduces to Eq. 1.4). This approach via BSDEs only requires quasi-integrability of $F(\cdot, u)$. It turns out that this mild demand is always satisfied for solutions of Eq. 1.1. Therefore in the present paper we use some suitable generalization of the definition from [27] (see Section 3).

As for "compactness properties", let us note that in [28] it is shown that if $N=1$ and $F$ is nonincreasing then for $A$ associated with a Dirichlet form the function $F(\cdot, u)$ is integrable but in general, $u$ is not integrable (even locally). Since in case $N \geq 2$ also the function $F(\cdot, u)$ need not be integrable, it is fairly unclear what type of function space possessing Banach structure to use to get the existence result for Eq. 1.1. In [27] we have used the specific structure of the operator $A=\Delta$ to prove that a solution of Eq. 1.1 equals locally (i.e. on some finely open sets) to some function from $H_{0}^{1}(D)$, which allowed us to apply the Rellich-Kondrachov theorem on finely open sets (see also [20, 21] for the theory of Laplacians on finely open domains). In general, this approach fails. To overcome the difficulty, in the present paper we introduce a notion of compactness property relating the process $\mathbb{X}$ to given solid $\mathcal{P}$ and positive subadditive set function $m$ on $E$ (not necessarily measure). The compactness property is intended to study $m$-a.e. convergence of sequences of functions defined on $E$, pointwise convergence (when $m$ is a counting measure) and quasi-everywhere convergence (when $m$ is the capacity determined by $A$ ). It appears that such analysis of pointwise behaviour of sequences of functions, in particular sequences of the form $\left\{p_{t} f_{n}\right\},\left\{R_{\alpha} f_{n}\right\}$, where $p_{t} f$ is defined by Eq. 1.2 and $R_{\alpha} f$ is the probabilistic resolvent defined by

$$
R_{\alpha} f(x)=E_{x} \int_{0}^{\infty} e^{-\alpha t} f\left(X_{t}\right) d t, \quad x \in E,
$$

is sufficient for the proof of existence of probabilistic solutions to Eq. 1.1.

Roughly speaking, given a solid $\mathcal{P} \subset \mathcal{B}^{+}(E)$ and a positive subadditive set function $m$ on $E$ we say the triple $(\mathbb{X}, \mathcal{P}, m)$ has the compactness property if for some $\alpha>0$ the probabilistic resolvent (1.5) maps the family $\mathcal{P}$ to a relatively compact set with respect to the topology of $m$-a.e. convergence (see Section 2.1). If $m$ is the counting measure then we will omit $m$ in the notation and simply say that $(\mathbb{X}, \mathcal{P})$ has the compactness property.

In applications the family $\mathcal{P}=\left\{u \in \mathcal{B}^{+}(E) ; u \leq 1\right\} \equiv \mathcal{B}_{1}$ plays pivotal role. From the well known results (see [17, Section IX, Theorem 16], [8, Lemma B, page 133]) it follows that if $\mathbb{X}$ satisfies hypothesis (L) of Meyer (see Section 2.2 for the definition) then $\left(\mathbb{X}, \mathcal{B}_{1}\right.$ ) has the compactness property. By the Mokobodzki theorem (see [8, Proposition 4.4.5]) and [36, Proposition 5.2]), $\mathbb{X}$ satisfies Meyer's hypothesis (L) if and only if there exists a strictly positive $\psi \in \mathcal{B}_{b}(E)$ such that $R_{\alpha}^{\psi}: \mathcal{B}_{b}(E) \rightarrow \mathcal{B}_{b}(E)$ defied as $R_{\alpha}^{\psi}(u)=R_{\alpha}(\psi u)$ for $u \in \mathcal{B}_{b}(E)$ is compact if we equip $\mathcal{B}_{b}(E)$ with the topology of uniform convergence. In Section 2 , using results of $[41,42]$, we prove that

$\left(\mathbb{X}, \mathcal{B}_{1}\right)$ has the compactness property iff $\mathbb{X}$ satisfies Meyer's hypothesis (L). 
In Section 4 we show that if $m$ is an excessive measure then

$$
\begin{aligned}
& \left(\mathbb{X}, \mathcal{B}_{1}, m\right) \text { has the compactness property iff } \\
& \qquad R_{\alpha}: L^{1}(E ; m) \rightarrow L^{1}(E ; m) \text { is order compact for some } \alpha>0 .
\end{aligned}
$$

Here by order compactness we mean that for every positive $v \in L^{1}(E ; m), R_{\alpha}$ carries order intervals $[0, v]=\left\{u \in L^{1}(E ; m): 0 \leq u \leq v\right\}$ in relatively compact subsets of $L^{1}(E ; m)$. We also investigate some stability properties of the compactness property with respect to transformation of the underlying process. The most important result in this direction is Proposition 2.8. It says that for every $B \in \mathcal{B}(E)$, if $(\mathbb{X}, \mathcal{P}, m)$ has the compactness property then $\left(\mathbb{X}^{B}, \mathcal{P}(B), m\right)$ has the compactness property, where $\mathbb{X}^{B}$ denotes the part of $\mathbb{X}$ on $B$ and $\mathcal{P}(B)=\{u \in \mathcal{P} ; u(x)=0, x \in E \backslash B\}$. We have already mentioned that it is reasonable to expect that $F(\cdot, u)$ and $u$ are quasi-integrable which roughly speaking means that they are integrable on subsets of $E$ whose complements have small capacity naturally generated by the operator $A$. The significance of Proposition 2.8 is that it allows to reduce the proof of existence of solutions of Eq. 1.1 to the analysis of the system (1.1) on such sets. Let us also note that in some sense Proposition 2.8 resembles results on compactness of positive operators subordinated to compact operator (see [2] and Corollary 2.10).

The second problem that we address in Section 2 is to find conditions on a sequence $\left\{u_{n}\right\}$ of functions on $E$, which together with the compactness property imply that $\left\{u_{n}\right\}$ is relatively compact in the topology of $m$-a.e. convergence. Our main result is Theorem 2.2, which says that if $(\mathbb{X}, \mathcal{P}, m)$ has the compactness property and $\left\{u_{n}\right\} \subset \mathcal{P}$ satisfies the condition

$$
\lim _{t \rightarrow 0^{+}} \sup _{n \geq 1}\left|p_{t} u_{n}(x)-u_{n}(x)\right|=0 \quad \text { for } m \text {-a.e. } x \in E
$$

then $\left\{u_{n}\right\}$ has a subsequence convergent $m$-a.e. Condition (1.7) is satisfied for instance if for $m$-a.e. $x \in E$ the sequence of processes $\left\{u_{n}(X)\right\}$ is tight in the Skorokhod topology $J_{1}$ under the measure $P_{x}$. It is worth noting here that in the paper the notion of compactness of a triple $(\mathbb{X}, \mathcal{P}, m)$ is defined for general normal processes (i.e. markovianity of the process $\mathbb{X}$ is not required) and that Theorem 2.2 is proved for such wide class of processes.

In Section 2.3 we show that if $\mathbb{X}$ is associated with a transient symmetric regular Dirichlet form $(\mathcal{E}, D[\mathcal{E}])$ on $L^{2}(E ; m),(\mathbb{X}, \mathcal{P}, m)$ has the compactness property and $\left\{u_{n}\right\} \subset \mathcal{F}_{e} \cap \mathcal{P}$, where $\mathcal{F}_{e}$ is an extension of the domain $D[\mathcal{E}]$ such that the pair $\left(\mathcal{E}, \mathcal{F}_{e}\right)$ is a Hilbert space, then the condition

$$
\sup _{n \geq 1} \mathcal{E}\left(u_{n}, u_{n}\right)<\infty
$$

implies that $\left\{u_{n}\right\}$ has a subsequence convergent $m$-a.e. Moreover, we prove that

$$
(\mathbb{X}, \mathcal{P}, m) \text { has the compactness property iff }
$$

$(\mathbb{X}, \mathcal{P}$, cap) has the compactness property,

where cap is the capacity on $E$ determined by the form $(\mathcal{E}, D[\mathcal{E}])$.

In Sections 3 and 4 we define a probabilistic solution of Eq. 1.1 and give an existence result for system (1.1). The basic space in which solutions are looked for is the space $\mathbf{D}$ of measurable functions $u$ on $E$ such that the family $\left\{u\left(X_{\tau}\right), \tau\right.$ is a stopping time $\}$ is uniformly integrable under $P_{x}$ for q.e. $x \in E$. We show that $D[\mathcal{E}] \subset \mathbf{D}$ if $\mathbb{X}$ is associated with a 
semi-Dirichlet form. We call a finely continuous function $u \in \mathbf{D}$ such that $F(\cdot, u)$ is quasiintegrable a solution of Eq. 1.1 if there exists a local martingale additive functional $M$ of $\mathbb{X}$ such that for $m$-a.e. $x \in E$ and every $T>0$,

$$
\begin{aligned}
u\left(X_{t}\right)= & u\left(X_{T \wedge \zeta}\right)+\int_{t}^{T \wedge \zeta} F\left(X_{r}, u\left(X_{r}\right)\right) d r+\int_{t}^{T \wedge \zeta} d A_{r}^{\mu} \\
& +\int_{t}^{T \wedge \zeta} d M_{r}, \quad t \in[0, T \wedge \zeta], \quad P_{x} \text {-a.s., }
\end{aligned}
$$

where $\zeta$ is the life-time of $\mathbb{X}$ and $A^{\mu}$ is the positive co-natural additive functional associated with measure $\mu$.

We first study probabilistic solutions to Eq. 1.1 in case $\mathbb{X}$ is associated with a semiDirichlet form and $\left(\mathbb{X}, \mathcal{B}_{1}, m\right)$ has the compactness property. In Section 3 we show that if $\mu$ is smooth and satisfies some integrabilty condition, $F$ satisfies the sign condition (1.3), then there exists a solution of Eq. 1.1. We also show that if $F$ is monotone, i.e.

$$
\langle F(x, y)-F(x, z), y-z\rangle \leq 0, \quad x \in E, y, z \in \mathbb{R}^{N},
$$

then the probabilistic solution to Eq. 1.1 is unique.

The case of general right Markov processes is considered in Section 4. We show that if $\mathbb{X}$ satisfies Meyer's condition (L) then under the same hypotheses as in Section 3 there exists a solution to Eq. 1.1. Using Eq. 1.6 one can formulate the existence result in purely analytic terms, without relating to the concept of the compactness property. Namely, if the resolvent of the operator $A$ is order compact on $L^{1}(E ; m), F$ satisfies the sign condition and the data are appropriately integrable then there exists a solution of Eq. 1.1. As a matter of fact we assume some additional regularity condition on the semigroup $\left\{T_{t}, t \geq 0\right\}$ but we think that it is technical and can be omitted.

In Section 5 we give some examples of operators and processes to which our results apply. Among others we give a simple example of Ornstein-Uhlenbeck semigroup, i.e. semigroup generated by differential operator of the form

$$
L \phi(x)=\frac{1}{2} \operatorname{tr}\left(Q D^{2} \phi(x)\right)+\langle A x, D \phi(x)\rangle,
$$

which is not of variational form (or, equivalently, is not analytic). The Ornstein-Uhlenbeck process with generator $L$ is not associated with a Dirichlet form but satisfies Meyer's hypothesis (L). This shows that the class of processes considered in Section 4 includes important processes that do not belong to the class considered in Section 3.

\section{Compactness Property}

\subsection{Normal Processes}

Let $E$ be a Radon metrizable topological space (see [7]) and $\mathcal{B}(E)$ be the set of all numerical Borel measurable functions on $E$. W adjoin an isolated point $\Delta$ to $E$ and set $E_{\Delta}=E \cup\{\Delta\}$ (in $E_{\Delta}$ we have natural topology in which $E$ is open). We denote by $\mathcal{B}_{\Delta}(E)$ the set of all numerical Borel measurable functions on $E_{\Delta}$. Let $(\Omega, \mathcal{G})$ be a measurable space and $\left\{X_{t}, t \in[0, \infty]\right\}$ be a stochastic process on $E_{\Delta}$ such that $X_{\infty}=\Delta$ and if $X_{t_{0}}=\Delta$ for some $t_{0} \in[0, \infty]$ then $X_{t}=\Delta$ for $t \geq t_{0}$. We denote by $\zeta$ the life-time of $X$, i.e.

$$
\zeta=\inf \left\{t \geq 0 ; \quad X_{t}=\Delta\right\} .
$$


For $x \in E_{\Delta}$ let $P_{x}$ be a probability measure on $(\Omega, \mathcal{G})$. Let $\left\{\mathcal{G}_{t}, t \in[0, \infty]\right\}$ be a filtration in $\mathcal{G}$ and let $\left\{\mathcal{G}_{t}^{0}, t \geq 0\right\}$ be the natural filtration generated by $X$. We assume that

(a) for every $t \geq 0, X_{t} \in \mathcal{G}_{t} / \mathcal{B}_{\Delta}(E)$,

(b) the mapping $E \ni x \mapsto P_{x}\left(X_{t} \in B\right)$ belongs to $\mathcal{B}(E)$ for every $t \geq 0$ and $B \in \mathcal{B}(E)$,

(c) for every $x \in E_{\Delta}, P_{x}\left(X_{0}=x\right)=1$,

(d) $X$ is measurable relative to $\mathcal{G}^{0}$, i.e. the mapping $[0, \infty) \times \Omega \ni(t, w) \mapsto X_{t}(w) \in E_{\Delta}$ is $\mathcal{B}([0, \infty)) \times \mathcal{G}^{0} / \mathcal{B}_{\Delta}$ measurable.

Let $\mathbb{X}=\left(\Omega, \mathcal{F},\left\{X_{t}, t \geq 0\right\},\left\{P_{x}, x \in E\right\}\right)$. In the whole paper for a given Borel set $B \subset E$ we denote by

$$
\sigma_{B}=\inf \left\{t>0 ; X_{t} \in B\right\}, \quad D_{B}=\inf \left\{t \geq 0 ; X_{t} \in B\right\}, \quad \tau_{B}=\inf \left\{t>0 ; X_{t} \in E \backslash B\right\}
$$

the hitting time, debut time and the first exist time of $B$, respectively. By $\mathbb{X}^{B}=$ $\left(\Omega, \mathcal{F},\left\{X_{t}^{B}, t \geq 0\right\},\left\{P_{x}, x \in E\right\}\right)$ we denote the part of $\mathbb{X}$ on $B$, i.e.

$$
X_{t}^{B}= \begin{cases}X_{t}(\omega), & 0 \leq t<D_{E \backslash B}(\omega), \\ \Delta, & t \geq D_{E \backslash B}(\omega) .\end{cases}
$$

Let $\mathcal{B}^{+}(E)=\{f \in \mathcal{B}(E) ; f(x) \geq 0, x \in E\}$ and let $\mathcal{B}^{r}(E)$ denote the set of $u \in \mathcal{B}(E)$ such that $u(x) \in \mathbb{R}$ for $x \in E$. In the whole paper we adopt the convention that $f(\Delta)=0$ for every numerical function $f$ on $E$. For every $t \geq 0, \alpha \geq 0$ and $f \in \mathcal{B}^{+}(E)$ we put

$$
p_{t} f(x)=E_{x} f\left(X_{t}\right), \quad R_{\alpha} f(x)=E_{x} \int_{0}^{\infty} e^{-\alpha t} f\left(X_{t}\right) d t, \quad x \in E .
$$

By (a)-(d), $p_{t}: \mathcal{B}^{+}(E) \rightarrow \mathcal{B}^{+}(E), R_{\alpha}: \mathcal{B}^{+}(E) \rightarrow \mathcal{B}^{+}(E)$. Let $\mathcal{P} \subset \mathcal{B}^{r,+}(E)$ be some family having the following properties

(P1) $\left(f \in \mathcal{P}, g \in \mathcal{B}^{+}(E), g \leq f\right) \Longrightarrow g \in \mathcal{P}$,

(P2) $\left\{f_{n}\right\} \subset \mathcal{P} \Longrightarrow \sup _{n} f_{n} \in \mathcal{P}$.

Unless otherwise stated, in this section $m$ is a nonnegative subadditive set function on $E$.

Definition (a) We say that a triple $(\mathbb{X}, \mathcal{P}, m)$ has the compactness property if for every $\left\{u_{n}\right\} \subset \mathcal{P}$ there exist a set $\Lambda \subset(0,+\infty)$ and a subsequence $\left(n^{\prime}\right) \subset(n)$ such that $\sup \Lambda=$ $+\infty$ and for every $\alpha \in \Lambda$ the sequence $\left\{R_{\alpha} u_{n^{\prime}}\right\}$ is $m$-a.e. convergent and its limit is $m$-a.e. finite.

(b) We say that a pair $(\mathbb{X}, \mathcal{P})$ has the compactness property if the triple $(\mathbb{X}, \mathcal{P}, m)$ has compactness property with $m$ being the counting measure.

In the sequel for given $\mathcal{P} \subset \mathcal{B}^{r,+}(E)$ we set $\mathcal{P}^{*}=\mathcal{P}-\mathcal{P}$.

Definition We say that a sequence $\left\{u_{n}\right\} \subset \mathcal{P}^{*}$ satisfies

(a) condition $\left(\mathrm{M}_{0}\right)$ if

$$
\lim _{h \rightarrow 0^{+}} \sup _{n \geq 1} \sup _{t \leq h}\left|u_{n}\left(X_{t}\right)-u_{n}(x)\right|=0, \quad P_{x} \text {-a.s. for } m \text {-a.e. } x \in E,
$$

(b) condition $\left(\mathrm{M}_{1}\right)$ if

$$
\lim _{t \rightarrow 0^{+}} \sup _{n \geq 1}\left|p_{t} u_{n}(x)-u_{n}(x)\right|=0 \quad \text { for } m \text {-a.e. } x \in E,
$$


(c) condition $\left(\mathrm{M}_{2}\right)$ if $m$ is a measure and for some $p \geq 1$

$$
\lim _{t \rightarrow 0^{+}} \sup _{n \geq 1}\left\|p_{t} u_{n}-u_{n}\right\|_{L^{p}(E ; m)}=0 .
$$

Remark 2.1 It is clear that if $\left\{u_{n}\right\}$ satisfies some integrability conditions and $m$ is a $\sigma$-finite measure then $\left(\mathrm{M}_{0}\right)$ implies $\left(\mathrm{M}_{1}\right)$ and $\left(\mathrm{M}_{1}\right)$ implies $\left(\mathrm{M}_{2}\right)$.

Theorem 2.2 Assume that $(\mathbb{X}, \mathcal{P}, m)$ has the compactness property. If $\left\{u_{n}\right\} \subset \mathcal{P}^{*}$ satisfies $\left(\mathrm{M}_{1}\right)$ then there exists a subsequence $\left(n^{\prime}\right) \subset(n)$ such that $\left\{u_{n^{\prime}}\right\}$ is $m$-a.e. convergent and its limit is $m$-a.e. finite.

Proof Let $\Lambda \subset(0,+\infty)$ be a countable set such that sup $\Lambda=+\infty$ and let $\left(n^{\prime}\right) \subset(n)$ be a subsequence such that for every $\alpha \in \Lambda,\left\{R_{\alpha} u_{n^{\prime}}\right\}$ is $m$-a.e. convergent and its limit is finite $m$-a.e. Let $A \subset E$ be a set of those $x \in E$ for which $\lim _{n^{\prime}} R_{\alpha} u_{n^{\prime}}(x)$ does not exist or exists and is infinite for some $\alpha \in \Lambda$. It is clear that $m(A)=0$. Let $B$ be the set of those $x \in E$ for which condition $\left(\mathrm{M}_{1}\right)$ does not hold. We put $w=\sup _{n}\left|u_{n}\right|$. By (P1) and (P2), $u_{n}^{+}, u_{n}^{-} \in \mathcal{P}$ and $\sup _{n} u_{n}^{+}, \sup _{n} u_{n}^{-} \in \mathcal{P}$. Since $w \leq \sup _{n} u_{n}^{+}+\sup _{n} u_{n}^{-}$, we see that $w(x)<\infty$ for $x \in E$ and that without loss of generality we may assume that $R_{\alpha} w(x)<\infty$ for $m$-a.e. $x \in E$ and every $\alpha \in \Lambda$. Let $C \subset E$ be the set of those $x \in E$ for which $R_{\alpha} w(x)=+\infty$ for some $\alpha \in \Lambda$. Let $N=A \cup B \cup C$. It is clear that $m(N)=0$. Let $x \in E \backslash N$. Then

$$
\left|\alpha R_{\alpha} u_{n}(x)-u_{n}(x)\right| \leq \alpha \int_{0}^{\infty} e^{-\alpha t}\left|p_{t} u_{n}(x)-u_{n}(x)\right| d t .
$$

Let us fix $\varepsilon>0$ and let $\theta_{x}^{\varepsilon}>0, n_{x}^{\varepsilon} \in \mathbb{N}$ be such that

$$
\sup _{t \leq \theta_{x}^{\varepsilon}}\left|p_{t} u_{n}(x)-u_{n}(x)\right|<\frac{\varepsilon}{2}, \quad n \geq n_{x}^{\varepsilon} .
$$

Then

$$
\begin{aligned}
& \alpha \int_{0}^{\infty} e^{-\alpha t}\left|p_{t} u_{n}(x)-u_{n}(x)\right| d t \\
& \quad \leq \alpha \int_{0}^{\theta_{x}^{\varepsilon}} e^{-\alpha t}\left|p_{t} u_{n}(x)-u_{n}(x)\right| d t+\alpha \int_{\theta_{x}^{\varepsilon}}^{\infty} e^{-\alpha t}\left|p_{t} u_{n}(x)-u_{n}(x)\right| d t \\
& \quad \leq \alpha \frac{\varepsilon}{2} \int_{0}^{\theta_{x}^{\varepsilon}} e^{-\alpha t} d t+\alpha \int_{\theta_{x}^{\varepsilon}}^{\infty} e^{-\alpha t} p_{t} w(x) d t+\alpha w(x) \int_{\theta_{x}^{\varepsilon}}^{\infty} e^{-\alpha t} d t \\
& \quad \leq \frac{\varepsilon}{2}\left(1-e^{-\alpha \theta_{x}^{\varepsilon}}\right)+w(x) e^{-\alpha \theta_{x}^{\varepsilon}}+\alpha \int_{\theta_{x}^{\varepsilon}}^{\infty} e^{-\alpha t} p_{t} w(x) d t .
\end{aligned}
$$

Let $\alpha_{0} \in \Lambda$ be such that $\alpha_{0}>1 / \theta_{x}^{\varepsilon}$. Then we have

$$
\alpha e^{-\alpha t} p_{t} w(x) \leq \alpha_{0} e^{-\alpha_{0} t} p_{t} w(x), \quad t \geq \theta_{x}^{\varepsilon}, \quad \alpha \geq \alpha_{0}, \quad \alpha \in \Lambda .
$$

Therefore there exists $\alpha_{x}^{\varepsilon} \in \Lambda$ such that

$$
\left|\alpha R_{\alpha} u_{n}(x)-u_{n}(x)\right|<\varepsilon, \quad n \geq n_{x}^{\varepsilon}, \quad \alpha \in \Lambda, \quad \alpha \geq \alpha_{x}^{\varepsilon} .
$$

Write $\beta_{x}=\lim \sup _{n^{\prime}} u_{n^{\prime}}(x)-\liminf _{n^{\prime}} u_{n^{\prime}}(x)$. Then there exists a subsequence $\left(n_{k}^{\prime}\right) \subset\left(n^{\prime}\right)$ such that

$$
\left|u_{n_{k+1}^{\prime}}(x)-u_{n_{k}^{\prime}}(x)\right|>\frac{\beta_{x}}{2}, \quad k \geq 1 .
$$


On the other hand, by Eq. 2.3, for $n_{k}^{\prime} \geq n_{x}^{\varepsilon}$ and $\alpha \in \Lambda$ such that $\alpha \geq \alpha_{x}^{\varepsilon}$,

$$
\begin{aligned}
\left|u_{n_{k+1}^{\prime}}(x)-u_{n_{k}^{\prime}}(x)\right| \leq & \left|u_{n_{k+1}^{\prime}}(x)-\alpha R_{\alpha} u_{n_{k+1}^{\prime}}(x)\right|+\left|\alpha R_{\alpha} u_{n_{k+1}^{\prime}}(x)-\alpha R_{\alpha} u_{n_{k}^{\prime}}(x)\right| \\
& +\left|\alpha R_{\alpha} u_{n_{k}^{\prime}}(x)-u_{n_{k}^{\prime}}(x)\right| \\
\leq & 2 \varepsilon+\left|\alpha R_{\alpha} u_{n_{k+1}^{\prime}}(x)-\alpha R_{\alpha} u_{n_{k}^{\prime}}(x)\right| .
\end{aligned}
$$

Put $\varepsilon=\beta_{x} / 9$. By the compactness property of the triple $(\mathbb{X}, \mathcal{P}, m)$ there exists $N\left(\varepsilon, x, \alpha_{x}^{\varepsilon}\right) \in \mathbb{N}$ such that

$$
\left|\alpha_{x}^{\varepsilon} R_{\alpha_{x}^{\varepsilon}} u_{n_{k+1}^{\prime}}(x)-\alpha_{x}^{\varepsilon} R_{\alpha_{x}^{\varepsilon}} u_{n_{k}^{\prime}}(x)\right|<\frac{\beta_{x}}{9}
$$

for $n_{k}^{\prime} \geq N\left(\varepsilon, x, \alpha_{x}^{\varepsilon}\right)$. By Eqs. 2.5 and 2.6,

$$
\left|u_{n_{k+1}^{\prime}}(x)-u_{n_{k}^{\prime}}(x)\right| \leq \frac{\beta_{x}}{3}
$$

for $n_{k}^{\prime} \geq \max \left\{n_{x}^{\varepsilon}, N\left(\varepsilon, x, \alpha_{x}^{\varepsilon}\right)\right\}$, which contradicts (2.4) and proves the theorem.

In what follows for $k \geq 0$ we put

$$
T_{k}(y)=\max \{\min \{y, k\},-k\}, \quad y \in \mathbb{R} .
$$

Corollary 2.3 Let $\left\{u_{n}\right\} \subset \mathcal{P}^{*}$. If $(\mathbb{X}, \mathcal{P}, m)$ has the compactness property and $\left\{u_{n}(X)\right\}$ is a sequence of càdlàg processes on some interval $[0, T]$ tight in the Skorokhod topology $J_{1}$ under the measure $P_{x}$ for $m$-a.e. $x \in E$ then $\left\{u_{n}\right\}$ has a subsequence convergent $m$-a.e.

Proof If $\left\{u_{n}(X)\right\}$ is tight under $P_{x}$ for $m$-a.e. $x \in E$ then condition $\left(\mathrm{M}_{0}\right)$ is satisfied for $m$-a.e. $x \in E$ and $\sup _{n \geq 1}\left|u_{n}(x)\right|$ is finite $m$-a.e. Of course the same is true for $\left\{u_{n}^{k}(X)\right\}$ for every $k \geq 0$, where $u_{n}^{k}=T_{k}\left(u_{n}\right)$. Observe that if $\left\{u_{n}^{k}\right\}$ satisfies $\left(\mathrm{M}_{0}\right)$ then it satisfies $\left(\mathrm{M}_{1}\right)$. Therefore from Theorem 2.2 it follows that $\left\{u_{n}^{k}\right\}$ converges $m$-a.e. up to a subsequence for every $k \geq 1$. From this we easily deduce that there exists a subsequence $\left(n^{\prime}\right) \subset(n)$ such that $\left\{u_{n^{\prime}}\right\}$ converges $m$-a.e.

\subsection{Right Markov Processes}

In this section we will show an equivalent condition to absolute continuity condition (the so called Meyer's hypothesis (L)) for $\mathbb{X}$ via the notion of compactness property. For other interesting conditions ensuring Meyer's hypothesis (L) for $\mathbb{X}$ see $[6,41]$.

Let us recall that $\mathbb{X}$ satisfies Meyer's hypothesis (L) if there exists a $\sigma$-finite Borel measure $m$ on $E$ such that $R_{\alpha}(x, d y) \ll m$ for every $x \in E$ and some (and hence every) $\alpha>0$.

The measure $m$ of the above definition will be called a reference measure for the process $\mathbb{X}$ or a reference measure for the resolvent $\left\{R_{\alpha}, \alpha>0\right\}$.

Let $\mathcal{B}_{1}=\left\{u \in \mathcal{B}^{+}(E) ; u(x) \leq 1, x \in E\right\}$

Proposition 2.4 Assume that $\mathbb{X}$ is a right Markov process. Then $\left(\mathbb{X}, \mathcal{B}_{1}\right)$ has the compactness property iff $\mathbb{X}$ satisfies Meyer's hypothesis (L).

Proof If $\mathbb{X}$ satisfies Meyer's hypothesis (L) then by [8, Lemma B, page 133], $\left(\mathbb{X}, \mathcal{B}_{1}\right)$ has the compactness property. Suppose now that $\left(\mathbb{X}, \mathcal{B}_{1}\right)$ has the compactness property. From the resolvent identity it is clear that if $R_{\alpha}$ has a reference measure for some $\alpha>0$ then 
$\mathbb{X}$ has a reference measure. Consequently, if $\mathbb{X}$ does not have a reference measure then for every $\alpha>0$ the resolvent $R_{\alpha}$ does not have a reference measure. In [42] it is proved that if $R_{\alpha}$ does not have a reference measure then there exists a compact perfect set $K \subset E$ such that $\mathbf{1}_{K} \cdot R_{\alpha}: \mathcal{B}_{b}(E) \rightarrow \mathcal{B}_{b}(K)$ is surjective. Moreover, from the proof in [42] it follows that there exists $\gamma>0$ such that for every $g \in \mathcal{B}_{b}^{+}(K)$ such that $\sup _{x \in E}|g(x)| \leq c$ for some $c>0$ there exists $f \in \mathcal{B}_{b}^{+}(E)$ such that $\left(\mathbf{1}_{K} R_{\alpha}\right) f=g$ and $|f(x)| \leq \gamma c$ for $x \in E$. Since $K$ is uncountable, there exists a sequence $\left\{v_{n}\right\} \subset \mathcal{B}_{b}^{+}(K)$ such that $\left|v_{n}(x)\right| \leq 1 / \gamma$ for $x \in E$, $n \geq 1$ and $\left\{v_{n}\right\}$ has no subsequence converging pointwise. Thanks to the properties of the operator $\mathbf{1}_{K} R_{\alpha}$, for every $n \geq 1$ there exists $u_{n} \in \mathcal{B}_{1}^{+}(E)$ such that $\mathbf{1}_{K} R_{\alpha} u_{n}=v_{n}$. This implies that there exists a sequence $\left\{u_{n}\right\} \subset \mathcal{B}_{1}^{+}(E)$ such that $\left\{R_{\alpha} u_{n}\right\}$ has no subsequence converging pointwise.

Remark 2.5 Let $\mathbb{X}$ be a Markov process and $m$ be its excessive measure. If for some $\alpha>0$, $R_{\alpha}$ maps a family $\mathcal{B}_{1}$ to a relatively compact set in the topology of $m$-a.e. convergence then $R_{\beta}$ has the same property for every $\beta>\alpha$. To see this, let us suppose that $\beta>\alpha$ and $\left\{u_{n}\right\} \subset \mathcal{B}_{1}$. Then there exists a subsequence $\left(n^{\prime}\right) \subset(n)$ such that $\left\{R_{\alpha} u_{n^{\prime}}\right\},\left\{R_{\alpha}\left(R_{\beta} u_{n^{\prime}}\right)\right\}$ are $m$-a.e. convergent and their limits are finite $m$-a.e., since $\left\{\beta R_{\beta} u_{n^{\prime}}\right\} \subset \mathcal{B}_{1}$. From this and the resolvent identity

$$
R_{\beta} u_{n^{\prime}}=R_{\alpha} u_{n^{\prime}}+(\alpha-\beta) R_{\alpha}\left(R_{\beta} u_{n^{\prime}}\right)
$$

it follows that $\left\{R_{\beta} u_{n^{\prime}}\right\}$ is $m$-a.e. convergent and its limit is finite $m$-a.e. Therefore if $\mathbb{X}$ is a Markov process then the compactness of the triple $\left(\mathbb{X}, \mathcal{B}_{1}, m\right)$ is equivalent to saying that for some $\alpha>0$ the operator $R_{\alpha}$ maps the family $\mathcal{B}_{1}$ to a relatively compact set with respect to the topology of $m$-a.e. convergence.

\subsection{Hunt Processes Associated with Dirichlet Forms}

Let $E$ be a locally compact separable metric space. In the rest of this section $\mathbb{X}$ is a Hunt process associated with a regular semi-Dirichlet form on $L^{2}(E ; m)$. Let us recall that a semi-Dirichlet form on $L^{2}(E ; m)$ is a bilinear form

$$
\mathcal{E}: D[\mathcal{E}] \times D[\mathcal{E}] \rightarrow \mathbb{R}
$$

defined on a dense linear subspace $D[\mathcal{E}]$ of $L^{2}(E ; m)$ satisfying the following conditions

(a) there exists $\alpha_{0} \geq 0$ such that $\mathcal{E}_{\alpha}(u, u) \geq 0$ for every $u \in D[\mathcal{E}]$ and $\alpha \geq \alpha_{0}$,

(b) there exists $K>0$ such that $|\mathcal{E}(u, v)| \leq K\left(\mathcal{E}_{\alpha_{0}}(u, u)\right)^{1 / 2}\left(\mathcal{E}_{\alpha_{0}}(v, v)\right)^{1 / 2}$ for every $u, v \in D[\mathcal{E}]$,

(c) $D[\mathcal{E}]$ equipped with the inner product $\mathcal{E}_{\alpha_{0}}^{(s)}(\cdot, \cdot)$, where $\mathcal{E}_{\alpha}^{(s)}(u, v)=\frac{1}{2}\left(\mathcal{E}_{\alpha}(u, v)+\right.$ $\left.\mathcal{E}_{\alpha}(v, u)\right)$, is a Hilbert space,

(d) for every $u \in D[\mathcal{E}]$ and $k \geq 0, u \wedge k \in D[\mathcal{E}]$ and $\mathcal{E}(u \wedge k, u \wedge k) \leq \mathcal{E}(u \wedge k, u)$.

A semi-Dirichlet form $(\mathcal{E}, D[\mathcal{E}])$ is called regular if there exists a set $\mathcal{C} \subset C_{0}(E) \cap D[\mathcal{E}]$ $\left(C_{0}(E)\right.$ is the set of all continuous functions on $E$ with compact suppport) such that $\mathcal{C}$ is dense in $D[\mathcal{E}]$ in the norm determined by $\mathcal{E}_{\alpha_{0}}$ and in $C_{0}(E)$ in the norm of uniform convergence.

It is well known that with every regular semi-Dirichlet form $(\mathcal{E}, D[\mathcal{E}])$ one can associate uniquely a Hunt process $\mathbb{X}$ (see [34, Section 3.3]).

A semi-Dirichlet form $(\mathcal{E}, D[\mathcal{E}])$ is called positive if (a) is satisfied with $\alpha_{0}=0$ and is called transient if the associated Hunt process $\mathbb{X}$ is transient, i.e. there exists a strictly positive Borel measurable function $f$ on $E$ such that $R f$ is finite $m$-a.e. It is known that if 
a semi-Dirichlet form $(\mathcal{E}, D[\mathcal{E}])$ is transient and positive then there exists an extension $\mathcal{F}_{e}$ of the domain $D[\mathcal{E}]$ such that $\left(\mathcal{F}_{e}, \mathcal{E}^{(s)}(\cdot, \cdot)\right)$ is a Hilbert space.

By cap we denote the capacity on subsets of $E$ naturally associated with $(\mathcal{E}, D[\mathcal{E}]$ ) (see [34, Section 2.1]). We say that some property holds quasi everywhere (q.e. for short) if it holds except for a set $N \subset E$ such that $\operatorname{cap}(N)=0$.

We say that an increasing sequence $\left\{F_{n}\right\}$ of closed subsets of $E$ is a nest if for every compact $K \subset E, \operatorname{cap}\left(K \backslash F_{n}\right) \rightarrow 0$ as $n \rightarrow \infty$.

We say that a Borel measure $\mu$ on $E$ is smooth if it charges no set of zero capacity and there exists a nest $\left\{F_{n}\right\}$ such that $|\mu|\left(F_{n}\right)<\infty, n \geq 1$.

It is well known (see [34, Section 4.1]) that for every smooth measure $\mu$ there exists a unique continuous additive functional $A^{\mu}$ of $\mathbb{X}$ in the Revuz duality with $\mu$.

In the whole paper for a positive smooth measure $\mu$ and $\alpha \geq 0$ we write

$$
\left(R_{\alpha} \mu\right)(x)=E_{x} \int_{0}^{\zeta} e^{-\alpha r} d A_{r}^{\mu} .
$$

Observe that if $f \in \mathcal{B}^{+}(E)$ then $R_{\alpha}(f \cdot m)=R_{\alpha} f$, where $R_{\alpha}$ is defined by Eq. 1.5. We also write $R=R_{0}$.

Lemma 2.6 Let $\left\{u_{n}\right\} \subset \mathcal{B}(E)$ be such that $R_{\alpha} w<\infty$ m-a.e., where $w=\sup _{n}\left|u_{n}\right|$. If $\left\{R_{\alpha} u_{n}\right\}$ is convergent $m$-a.e. then there exists a subsequence $\left(n^{\prime}\right) \subset(n)$ such that $\left\{R_{\alpha} u_{n^{\prime}}\right\}$ is convergent q.e.

Proof Let $\left\{g_{k}\right\}$ be a sequence of Borel measurable functions on $E$ such that $0 \leq g_{k}(x) \leq 1$, $g_{k}(x) \nearrow 1$ for $x \in E$ and $g_{k} \cdot w \in L^{2}(E ; m)$ for every $k \geq 1$. Write $u_{n}^{k}=g_{k} u_{n}, v_{n}^{k}=$ $R_{\alpha}\left(u_{n}^{k}\right), v_{n}=R_{\alpha}\left(u_{n}\right)$. Then

$$
\left|v_{n}^{k}(x)-v_{n}(x)\right| \leq R_{\alpha}\left(w\left|1-g_{k}\right|\right)(x), \quad x \in E .
$$

Let $B=\left\{\inf _{k} R_{\alpha} w\left|1-g_{k}\right|>0\right\}$ and let $K$ be a compact set such that $K \subset B$. Then

$$
\begin{aligned}
P_{m}\left(\sigma_{K}<\infty\right)= & P_{m}\left(\sigma_{K}<\infty, \inf _{k \geq 1} E_{x}\left(\int_{\sigma_{K}}^{\infty} w\left|1-g_{k}\right|\left(X_{r}\right) d r \mid \mathcal{F}_{\sigma_{K}}\right)>0\right) \\
& \leq P_{m}\left(\sigma_{K}<\infty, \inf _{k \geq 1} E_{x}\left(\int_{0}^{\infty} w\left|1-g_{k}\right|\left(X_{r}\right) d r \mid \mathcal{F}_{\sigma_{K}}\right)>0\right)=0
\end{aligned}
$$

since $\inf _{k} R_{\alpha}\left(w\left|1-g_{k}\right|\right)(x)=0$ for $m$-a.e. $x \in E$. From this and Eq. 2.8 we conclude that

$$
\lim _{k \rightarrow \infty} \sup _{n \geq 1}\left|v_{n}^{k}(x)-v_{n}(x)\right|=0 \quad \text { for q.e. } x \in E .
$$

Therefore to prove the lemma it suffices to show that for every $k \geq 0,\left\{v_{n}^{k}\right\}$ is convergent q.e. But this follows immediately from the inequality

$$
\begin{aligned}
\mathcal{E}_{\alpha}\left(R_{\alpha} u_{n}^{k}-R_{\alpha} u_{m}^{k}, R_{\alpha} u_{n}^{k}-R_{\alpha} u_{m}^{k}\right) & =\left(u_{n}^{k}-u_{m}^{k}, R_{\alpha} u_{n}^{k}-R_{\alpha} u_{m}^{k}\right)_{L^{2}(E ; m)} \\
& \leq 2\left\|g_{k} \cdot w\right\|_{L^{2}(E ; m)} \cdot\left\|v_{n}^{k}-v_{m}^{k}\right\|_{L^{2}(E ; m)}
\end{aligned}
$$

and [34, Theorem 2.2.5]. 
Corollary 2.7 A triple $(\mathbb{X}, \mathcal{P}, m)$ has the compactness property iff $(\mathbb{X}, \mathcal{P}$, cap) has the compactness property.

For $B \in \mathcal{B}(E)$ set

$$
\mathcal{P}(B)=\{u \in \mathcal{B}(E) ; u(x)=0, x \in E \backslash B\}
$$

and for $\alpha \geq 0$ and $u \in \mathcal{B}^{+}(E)$ set

$$
R_{\alpha}^{E \backslash B} u(x)=E_{x} \int_{0}^{\sigma_{B}} e^{-\alpha t} u\left(X_{t}\right) d t, \quad H_{B}^{\alpha} u(x)=E_{x} e^{-\alpha \sigma_{B}} u\left(X_{\sigma_{B}}\right) .
$$

Proposition 2.8 Let $B \in \mathcal{B}(E)$. If $(\mathbb{X}, \mathcal{P}, m)$ has the compactness property then $\left(\mathbb{X}^{B}, \mathcal{P}(B), m\right)$ has the compactness property.

Proof Let $\left\{u_{n}\right\} \subset \mathcal{P}^{+}(B)$. By the assumption there exists a set $\Lambda \subset(0,+\infty)$ such that $\sup \Lambda=+\infty$ and a subsequence $\left(n^{\prime}\right) \subset(n)$ such that for every $\alpha \in \Lambda$ the sequence $\left\{R_{\alpha} u_{n^{\prime}}\right\}$ is convergent $m$-a.e. and its limit is finite $m$-a.e. By Dynkin's formula,

$$
R_{\alpha} u_{n^{\prime}}=R_{\alpha}^{B} u_{n^{\prime}}+H_{E \backslash B}^{\alpha}\left(R_{\alpha} u_{n^{\prime}}\right), \quad m \text {-a.e. }
$$

Therefore it suffices to show that up to a subsequence, $\left\{H_{E \backslash B}^{\alpha}\left(R_{\alpha} u_{n^{\prime}}\right)\right\}$ is $m$-a.e. convergent and its limit is finite $m$-a.e. But this follows immediately from Lemma 2.6 , because q.e. convergence of $\left\{R_{\alpha} u_{n^{\prime}}\right\}$ implies that $\left\{e^{-\alpha \tau_{B}} R_{\alpha} u_{n^{\prime}}\left(X_{\tau_{B}}\right)\right\}$ is convergent $P_{x}$-a.s. for $m$-a.e. $x \in E$, moreover we have $\left|R_{\alpha} u_{n}\right| \leq R_{\alpha} w, m$-a.e., where $w=\sup _{n}\left|u_{n}\right| \in \mathcal{P}$. Therefore we can apply the Lebesgue dominated convergence theorem to sequence $\left\{e^{-\alpha \tau_{B}} R_{\alpha} u_{n^{\prime}}\left(X_{\tau_{B}}\right)\right\}$ , because $\left\{\left|R_{\alpha} u_{n}\right|>R_{\alpha} w\right\}$ as a finely open $m$-negligible set is exceptional, which in turn implies that $\left|R_{\alpha} u_{n}\right| \leq R_{\alpha} w$ q.e., hence that $\left|\left(R_{\alpha} u_{n}\right)\right|\left(X_{\tau_{B}}\right) \leq\left(R_{\alpha} w\right)\left(X_{\tau_{B}}\right), P_{x}$-a.s. for $m$-a.e. $x \in E$ and $E_{x} R_{\alpha} w\left(X_{\tau_{B}}\right)=E_{x} \int_{\tau_{B}}^{\infty} e^{-\alpha r} w\left(X_{r}\right) d r \leq E_{x} \int_{0}^{\infty} e^{-\alpha r} w\left(X_{r}\right) d r=$ $R_{\alpha} w(x)<\infty$ for every $x \in E$.

Remark 2.9 Observe that the assertion of Proposition 2.8 holds true if we replace the process $\mathbb{X}^{B}$ killed outside a Borel set $B$ by the process $\mathbb{X}^{A}$ killed with rate $-d L_{t} / L_{t}$, where $L_{t}=e^{-A_{t}}$ for some positive continuous additive functional $A$ of $\mathbb{X}$ (for notation see [23, Theorem A.2.11]). To see this it suffices to repeat the proof of Proposition 2.8 with $\tau_{B}$ replaced by the stopping time

$$
\zeta_{A}=\inf \left\{t<\zeta ; A_{t} \geq Z\right\}
$$

where $Z$ is a random variable of exponential distribution with mean 1 independent of $\mathbb{X}$ and satisfying $Z\left(\theta_{s}(\omega)\right)=(Z(\omega)-s) \vee 0$.

Let us recall that a Markov process $\mathbb{X}^{0}$ on $E_{0} \in \mathcal{B}(E)$ is called a subprocess of $\mathbb{X}$ if its semigroup $\left\{p_{t}^{0}, t \geq 0\right\}$ extends naturally to $E$ subordinate to $\left\{p_{t}, t \geq 0\right\}$, i.e. for every $f \in \mathcal{B}^{+}(E)$ and $t \geq 0$,

$$
\bar{p}_{t}^{0} f \leq p_{t} f
$$

where $\bar{p}_{t}^{0} f(x)=p_{t}^{0} f_{\left.\right|_{E_{0}}}(x)$ for $x \in E_{0}$ and $\bar{p}_{t}^{0} f(x)=0$ for $x \in E \backslash E_{0}$, and the mapping $t \mapsto \bar{p}^{0} 1(x)$ is rightcontinuous at 0 for every $x \in E$.

Corollary 2.10 Let $\mathbb{X}^{0}$ be a subprocess of $\mathbb{X}$. If $(\mathbb{X}, \mathcal{P}, m)$ has the compactness property then $\left(\mathbb{X}^{0}, \mathcal{P}, m\right)$ has the compactness property. 
Proof By [11, Theorem III.2.3, page 101], $\mathbb{X}^{0}$ is equivalent to the process $\mathbb{X}$ killed with rate $-d L_{t} / L_{t}$, where $L$ is some right continuous multiplicative functional of $\mathbb{X}$. By similar construction as in Remark 2.9 one can show that the process $\mathbb{X}^{0}$ is in fact the killed process $\mathbb{X}$ at a terminal time constructed via multiplicative functional $L$ (see [37, Remark 2.1]), so the result, by the same argument as in Remark 2.9, follows from Proposition 2.8.

Let us consider the following additional condition on $\mathcal{E}$ (see [34, page 25]).

(f) If $w \in L^{2}(E ; m)$ and for some bounded $u, v \in D[\mathcal{E}]$ we have $|w(x)-w(y)| \leq|v(x)-v(y)|+|u(x)-u(y)|, \quad|w(x)| \leq|v(x)|+|w(x)|, \quad x, y \in E$, then $w \in D[\mathcal{E}]$ and there exists $K_{0}>0$ depending on $\|u\|_{\infty},\|v\|_{\infty}$ such that

$$
|\mathcal{E}(w, w)| \leq K_{0}\left(\mathcal{E}_{\alpha_{0}}(u, u)+\mathcal{E}_{\alpha_{0}}(v, v)\right) .
$$

Proposition 2.11 Assume that $\mathcal{E}$ is positive, satisfies condition (f) and $(\mathbb{X}, \mathcal{P}, m$ ) has the compactness property. If $\left\{u_{n}\right\} \subset \mathcal{F}_{e} \cap \mathcal{P}^{*}$ and

$$
\sup _{n \geq 1} \mathcal{E}\left(u_{n}, u_{n}\right)<\infty
$$

then there exists a subsequence $\left(n^{\prime}\right) \subset(n)$ such that $\left\{u_{n^{\prime}}\right\}$ is $m$-a.e. convergent and its limit is m-a.e. finite.

Proof Let $\eta \in D[\mathcal{E}]$ be such that $\eta>0, m$-a.e. and $\|\eta\|_{\infty}<\infty$. By (f),

$$
\mathcal{E}\left(T_{k}\left(u_{n}\right) \eta, T_{k}\left(u_{n}\right) \eta\right) \leq\|\eta\|_{\infty} \mathcal{E}\left(u_{n}, u_{n}\right)+k \mathcal{E}(\eta, \eta) .
$$

Hence

$$
\sup _{n} \mathcal{E}\left(T_{k}\left(u_{n}\right) \eta, T_{k}\left(u_{n}\right) \eta\right)<\infty .
$$

We can assume that $u_{n} \geq 0, m$-a.e. for every $n \geq 1$, because from (P1) it follows that $u_{n}^{+} \in \mathcal{P}$ and from [34, Eq. (1.1.12)] it follows that $u_{n}^{+} \in \mathcal{F}_{e}$ and $\mathcal{E}\left(u_{n}^{+}, u_{n}^{+}\right) \leq \mathcal{E}\left(u_{n}, u_{n}\right)$. Under the assumption of nonnegativity of $u_{n}, u_{n}^{k} \equiv T_{k}\left(u_{n}\right) \cdot \eta \in \mathcal{F}_{e} \cap \mathcal{P} \cap L^{2}(E ; m)$. By an elementary calculus,

$$
\left\|\alpha R_{\alpha} u_{n}^{k}-u_{n}^{k}\right\|_{L^{2}(E ; m)} \leq \alpha^{-1} \mathcal{E}\left(u_{n}^{k}, u_{n}^{k}\right),
$$

which when combined with Eq. 2.11 gives

$$
\left\|\alpha R_{\alpha} u_{n}^{k}-u_{n}^{k}\right\|_{L^{2}(E ; m)} \leq \alpha^{-1} c(k, \eta) .
$$

By the assumption there exists a subsequence $\left(n^{\prime}\right) \subset(n)$ and subset $\Lambda \subset(0, \infty)$ such that $\sup \Lambda=+\infty$ and $\left\{\alpha R_{\alpha} u_{n^{\prime}}^{k}\right\}$ is convergent in $L^{2}(E ; m)$ for every $\alpha \in \Lambda$. This and Eq. 2.12 imply that there exists a further subsequence $\left(n^{\prime \prime}\right) \subset\left(n^{\prime}\right)$ such that $\left\{u_{n^{\prime \prime}}^{k}\right\}$ is convergent in $L^{2}(E ; m)$. From this it follows easily that $\left\{u_{n^{\prime \prime \prime}}\right\}$ is convergent $m$-a.e. for some further subsequence $\left(n^{\prime \prime \prime}\right) \subset\left(n^{\prime \prime}\right)$.

Remark 2.12 If $\left\{u_{n}\right\} \subset \mathcal{F}_{e}$ satisfies (2.10) then by the calculations in the proof of [23, Lemma 1.5.4.] it satisfies condition $\left(\mathrm{M}_{2}\right)$.

In the sequel by $\mathcal{T}$ we denote the set of all stopping times to given filtration $\mathcal{F}$. 
Definition We say that a Borel measurable function $u$ on $E$ is of class (FD) if for $m$-a.e. $x \in E$ the family $\left\{u\left(X_{\tau}\right), \tau \in \mathcal{T}\right\}$ is uniformly integrable under the measure $P_{x}$.

By $\mathbf{D}$ we denote the set of all Borel measurable functions on $E$ of class (FD).

Remark 2.13 (i) Observe that $D[\mathcal{E}] \subset \mathbf{D}$. Indeed, each positive $u \in D[\mathcal{E}]$ is majorized by the $\alpha$-potential $e_{u}^{\alpha}$ (the smallest $\alpha$-potential majorizing $u$ ) and $e_{u}^{\alpha}=R_{\alpha} \mu$ for some measure $\mu$ of finite energy integral (see [34, Theorem 2.3.1]). Therefore by [34, Theorem 4.1.10],

$$
e_{u}^{\alpha}(x)=E_{x} \int_{0}^{\infty} e^{-\alpha t} d A_{t}^{\mu}
$$

From the above formula we easily deduce that $e_{u}^{\alpha} \in \mathbf{D}$ which implies that $u \in \mathbf{D}$. Since $u^{+}, u^{-} \in D[\mathcal{E}]$ if $u \in D[\mathcal{E}]$, we get the result.

(ii) If we assume additionally that $\mathcal{E}$ is positive and transient then in the same manner as in (i) we can show that $\mathcal{F}_{e} \subset \mathbf{D}$.

For $\alpha \geq 0$ and $\rho \in \mathcal{B}(E)$ such that $\rho>0$ let us define the space

$$
\mathbf{D}_{\alpha}=\left\{u \in \mathbf{D} ;\|u\|_{\alpha}<\infty\right\}
$$

where

$$
\|u\|_{\alpha}=\int_{E} \sup _{\tau \in \mathcal{T}} E_{x} e^{-\alpha \tau}\left|u\left(X_{\tau}\right)\right| \rho(x) m(d x) .
$$

In the sequel for a given $v \in \mathcal{B}^{+}(E)$ we write

$$
[0, v]=\{u \in \mathcal{B}(E) ; 0 \leq u \leq v\} .
$$

Proposition 2.14 Let $(\mathcal{E}, D[\mathcal{E}])$ be a regular symmetric Dirichlet form and $\mathbb{X}$ be a Hunt process associated with $(\mathcal{E}, D[\mathcal{E}])$. Then $\left(\mathbb{X}, \mathcal{B}_{1}, m\right)$ has the compactness property iff for every $\alpha>0$ the mapping $R_{\alpha}: \mathbf{D}_{0} \rightarrow \mathbf{D}_{\alpha}$ is order compact.

Proof Assume that $\left(\mathbb{X}, \mathcal{B}_{1}, m\right)$ has the compactness property. Let $v \in \mathbf{D}_{0}$ and $\left\{u_{n}\right\} \subset[0, v]$. Let $\left\{g_{k}\right\}$ be a sequence of positive Borel measurable functions on $E$ such that $g_{k} \nearrow 1$ as $k \rightarrow \infty$ and $g_{k} \cdot v \in L^{2}(E ; m)$. Put $u_{n}^{k}=g_{k} T_{k}\left(u_{n}\right), v_{n}^{k}=R_{\alpha}\left(u_{n}^{k}\right), v_{n}=R_{\alpha}\left(u_{n}\right)$. Then

$$
\begin{aligned}
\mathcal{E}_{\alpha}\left(R_{\alpha} u_{n}^{k}-R_{\alpha} u_{m}^{k}, R_{\alpha} u_{n}^{k}-R_{\alpha} u_{m}^{k}\right) & =\left(u_{n}^{k}-u_{m}^{k} ; R_{\alpha} u_{n}^{k}-R_{\alpha} u_{m}^{k}\right) \\
& \leq 2\left\|g_{k} \cdot v\right\|_{L^{2}(E ; m)} \cdot\left\|v_{n}^{k}-v_{m}^{k}\right\|_{L^{2}(E ; m)} .
\end{aligned}
$$

By the assumption, without loss of generality we may assume that for every $k \in \mathbb{N}$ the sequence $\left\{v_{n}^{k}\right\}$ is $m$-a.e. convergent as $n \rightarrow \infty$. Since $v_{n}^{k} \leq R_{\alpha}\left(g_{k} \cdot v\right) \in L^{2}(E ; m),\left\{v_{n}^{k}\right\}$ converges in $L^{2}(E ; m)$, and hence, by Eq. 2.13, in $\mathcal{E}_{\alpha}$. By [23, Lemma 5.1.1] this implies that there exists a subsequence (still denoted by $n$ ) such that for q.e. $x \in E$,

$$
\lim _{n, m \rightarrow \infty} E_{x} \sup _{t \geq 0} e^{-\alpha t}\left|v_{n}^{k}\left(X_{t}\right)-v_{m}^{k}\left(X_{t}\right)\right|=0 .
$$

Hence

$$
\lim _{n, m \rightarrow \infty} \sup _{\tau \in \mathcal{T}} E_{x} e^{-\alpha \tau}\left|v_{n}^{k}\left(X_{\tau}\right)-v_{m}^{k}\left(X_{\tau}\right)\right|=0
$$


for q.e. $x \in E$. By the Lebesgue dominated convergence theorem, $\left\|v_{n}^{k}-v_{m}^{k}\right\|_{\alpha} \rightarrow 0$ as $n, m \rightarrow \infty$, so it is enough to show that $\left\|v_{n}^{k}-v_{n}\right\|_{\alpha} \leq C(k)$ for some $C(k)$ such that $C(k) \rightarrow 0$ as $k \rightarrow \infty$. To this end, let us observe that

$$
\begin{aligned}
\left\|v_{n}^{k}-v_{n}\right\|_{\alpha} \leq & \int_{E} \sup _{\tau \in \mathcal{T}} E_{x} e^{-\alpha \tau}\left|R_{\alpha} u_{n}^{k}\left(X_{\tau}\right)-R_{\alpha} u_{n}\left(X_{\tau}\right)\right| m(d x) \\
\leq & \int_{E} \sup _{\tau \in \mathcal{T}} E_{x}\left(e^{-\alpha \tau} E_{x}\left(\int_{\tau}^{\infty} e^{-\alpha(r-\tau)}\left|u_{n}^{k}\left(X_{r}\right)-u_{n}\left(X_{r}\right)\right| d r \mid \mathcal{F}_{\tau}\right)\right) m(d x) \\
\leq & \int_{E} E_{x} \int_{0}^{\infty} e^{-\alpha r}\left|u_{n}^{k}\left(X_{r}\right)-u_{n}\left(X_{r}\right)\right| d r m(d x) \\
\leq & \int_{E} E_{x} \int_{0}^{\infty} e^{-\alpha r}\left|g_{k} T_{k}\left(u_{n}\right)-u_{n}\right|\left(X_{r}\right) d r m(d x) \\
\leq & \tau \int_{E} E_{x} \int_{0}^{\infty} e^{-\alpha r} \mathbf{1}_{\{v>k\}} v\left(X_{r}\right) m(d x) \\
& +\int_{E} E_{x} \int_{0}^{\infty} e^{-\alpha r}\left(v\left|g_{k}-1\right|\right)\left(X_{r}\right) d r m(d x) \equiv C(k) .
\end{aligned}
$$

Since $v \in \mathbf{D}_{0}$, both integrals on the right-hand side of last inequality are finite. Therefore by the Lebesgue dominated convergence theorem, $C(k) \rightarrow 0$ as $k \rightarrow \infty$, which shows the "if" part. Now, assume that $R_{\alpha}: \mathbf{D}_{0} \rightarrow \mathbf{D}_{\alpha}$ is order compact. Let $\left\{u_{n}\right\} \subset \mathcal{B}^{+}(E)$ be such that $u_{n}(x) \leq 1$ for $x \in E$. It is clear that $1 \in \mathbf{D}_{0}$ and $\left\{u_{n}\right\} \subset[0,1]$, so by order compactness of $R_{\alpha}: \mathbf{D}_{0} \rightarrow \mathbf{D}_{\alpha}$ it follows that there exists a subsequence (still denoted by $n$ ) such that

$$
\lim _{n, m \rightarrow \infty}\left\|R_{\alpha} u_{n}-R_{\alpha} u_{m}\right\|_{\alpha}=0 .
$$

In particular $\left\|R_{\alpha} u_{n}-R_{\alpha} u_{m}\right\|_{L^{1}(E ; \rho \cdot m)} \rightarrow 0$ as $n, m \rightarrow \infty$ from which we conclude that $\left(\mathbb{X}, \mathcal{B}_{1}, m\right)$ has the compactness property.

\section{Elliptic Systems with Measure Data on Dirichlet Space}

In this section we assume that $(\mathcal{E}, D[\mathcal{E}])$ is a transient regular semi-Dirichlet form on $L^{2}(E ; m)$. By $\mathbb{X}$ we denote a Hunt process associated with $(\mathcal{E}, D[\mathcal{E}])$.

In the sequel we adopt the convention that an $N$-dimensional process $Y$ or function $u$ has some property defined for one-dimensional processes or functions (for instance $Y$ is a MAF or CAF of $\mathbb{X}, u$ is of class (FD) etc.) if its each coordinate has this property.

Definition We say that a Borel measurable function $f$ on $E$ is quasi-integrable if for q.e. $x \in E$,

$$
P_{x}\left(\int_{0}^{\zeta \wedge T}\left|f\left(X_{r}\right)\right| d r<\infty, T>0\right)=1 .
$$

By $q L^{1}(E ; m)$ we denote that set of all quasi-integrable functions on $E$.

Remark 3.1 In the literature one can find another definition of quasi-integrability which we call here quasi-integrability in the analytic sense. According to this definition a measurable function $f$ on $E$ is quasi-integrable if for every $\varepsilon>0$ there exists an open set $U_{\varepsilon} \subset E$ such that $\operatorname{cap}\left(U_{\varepsilon}\right)<\varepsilon$ and $\left.f\right|_{E \backslash U_{\varepsilon}} \in L^{1}\left(E \backslash U_{\varepsilon} ; m\right)$. In [28] it is proved that if $f$ is quasi-integrable in the analytic sense then it is quasi-integrable. 
Let $F: E \times \mathbb{R}^{N} \rightarrow \mathbb{R}^{N}$ be a measurable function and $\mu=\left(\mu_{1}, \ldots, \mu_{N}\right)$ be a Borel measure on $E$ such that

(H1) $\quad \mu_{i}$ is a smooth measure such that $R\left|\mu_{i}\right|<\infty$ q.e.,

(H2) for every $r \geq 0$ the mapping $x \mapsto \sup _{|y| \leq r}|F(x, y)|$ belongs to $q L^{1}(E ; m)$,

(H3) for every $x \in E$ the mapping $y \mapsto F(x, y)$ is continuous,

(H4) there exists a non-negative function $G$ such that $R G<\infty$ q.e. and for every $x \in E$ and $y \in \mathbb{R}^{N}$,

$$
\langle F(x, y), y\rangle \leq G(x)|y| .
$$

We say that a real process $M$ is a local martingale additive functional (local MAF) of $\mathbb{X}$ if it is an additive functional of $\mathbb{X}$ (see [23, Section 5.1]) and $M$ is a local martingale under $P_{x}$ (with respect to the filtration $\mathcal{F}$ ) for each $x \in E \backslash N$, where $N$ is an exceptional set of $M$.

We would like to emphasize that the notion of local MAF differs from the notion of MAF locally of finite energy considered in [23, Section 5.5]. For instance, $M$ having the latter property is local AF, i.e. is additive on $[0, \zeta)$ only.

Let us consider the following system

$$
-A u=F(x, u)+\mu .
$$

Definition We say that a function $u: E \rightarrow \mathbb{R}^{N}$ is a solution of Eq. 3.1 if

(a) $u$ is quasi-continuous and $u \in \mathbf{D}$,

(b) $u\left(X_{t \wedge \zeta}\right) \rightarrow 0$ as $t \rightarrow \infty P_{x}$-a.s. for q.e. $x \in E$,

(c) $E \ni x \mapsto F(x, u(x)) \in q L^{1}(E ; m)$,

(d) there exists a local ( $N$-dimensional) MAF $M$ of $\mathbb{X}$ such that for q.e. $x \in E$ and every $T>0$,

$$
\begin{aligned}
u\left(X_{t}\right)= & u\left(X_{T \wedge \zeta}\right)+\int_{t}^{T \wedge \zeta} F\left(X_{r}, u\left(X_{r}\right)\right) d r+\int_{t}^{T \wedge \zeta} d A_{r}^{\mu} \\
& -\int_{t}^{T \wedge \zeta} d M_{r}, \quad t \in[0, T \wedge \zeta], \quad P_{x} \text {-a.s. }
\end{aligned}
$$

Remark 3.2 Observe that if $u: E \rightarrow \mathbb{R}^{N}$ is a measurable function such that $E_{x} \int_{0}^{\zeta}\left|F\left(X_{r}, u\left(X_{r}\right)\right)\right| d r<\infty$ and

$$
u(x)=E_{x} \int_{0}^{\zeta} F\left(X_{r}, u\left(X_{r}\right)\right) d r+E_{x} \int_{0}^{\zeta} d A_{r}^{\mu}
$$

for q.e. $x \in E$, then $u$ is a solution of Eq. 3.1. Indeed, by the Markov property,

$$
u\left(X_{t}\right)=E_{x}\left(\int_{t}^{\zeta} F\left(X_{r}, u\left(X_{r}\right)\right) d r+\int_{t}^{\zeta} d A_{r}^{\mu} \mid \mathcal{F}_{t}\right), \quad t \in[0, \zeta] .
$$

From this it is easily seen that $u \in \mathbf{D}$ and $u$ satisfies (b). It is also clear that (c) is satisfied. That $u$ is quasi-continuous it follows from [28, Lemma 4.2]. Now, let us put

$$
M_{t}^{x}=E_{x}\left(\int_{0}^{\zeta} F\left(X_{r}, u\left(X_{r}\right)\right) d r+\int_{0}^{\zeta} d A_{r}^{\mu} \mid \mathcal{F}_{t}\right)-u\left(X_{0}\right), \quad t \geq 0 .
$$

By [23, Lemma A.3.5] there exists a càdlàg process $M$ such that

$$
P_{x}\left(M_{t}=M_{t}^{x}, t \geq 0\right)=1
$$

for q.e. $x \in E$. It is clear that $M$ is a MAF of $\mathbb{X}$ and (d) is satisfied. 
We first show that if $F$ is monotone, i.e. $F$ satisfies the condition

$$
\left\langle F(x, y)-F\left(x, y^{\prime}\right), y-y^{\prime}\right\rangle \leq 0, y, y^{\prime} \in \mathbb{R}^{N}, x \in E,
$$

then the probabilistic solution of Eq. 3.1 is unique.

In the sequel for a given $x \in \mathbb{R}^{N}$ such that $x \neq 0$ we write

$$
\operatorname{sgn}(x)=\frac{x}{|x|} \text {. }
$$

Proposition 3.3 Assume that (H5) holds. Then the solution of Eq. 3.1 is q.e. unique.

Proof Let $u_{1}, u_{2}$ be solutions of Eq. 3.1 and $M_{1}, M_{2}$ be local MAFs associated with $u_{1}, u_{2}$, respectively. Put $u=u_{1}-u_{2}$ and $M=M_{1}-M_{2}$. Then

$u\left(X_{t}\right)=u\left(X_{\tau \wedge \zeta}\right)+\int_{t}^{\tau \wedge \zeta}\left(F\left(\cdot, u_{1}\right)-F\left(\cdot, u_{2}\right)\right)\left(X_{r}\right) d r-\int_{t}^{\tau \wedge \zeta} d M_{r}, \quad 0 \leq t \leq \tau \wedge \zeta, P_{x}$-a.s.

for every bounded $\tau \in \mathcal{T}$ and q.e. $x \in E$. By [9, Theorem 3] and (H5), for q.e. $x \in E$ we have

$$
\begin{aligned}
\left|u\left(X_{t}\right)\right| \leq & \left|u\left(X_{\tau \wedge \zeta}\right)\right|+\int_{t}^{\tau \wedge \zeta}\left\langle F\left(\cdot, u_{1}\right)-F\left(\cdot, u_{2}\right)\left(X_{r}\right), \operatorname{sgnn}\left(u\left(X_{r}\right)\right)\right\rangle d r \\
& -\int_{t}^{\tau \wedge \zeta}\left\langle\operatorname{sgnn}\left(u(X)_{r-}\right), d M_{r}\right\rangle \\
\leq & \left|u\left(X_{\tau \wedge \zeta}\right)\right|-\int_{t}^{\tau \wedge \zeta}\left\langle\operatorname{sgnn}\left(u(X)_{r-}\right), d M_{r}\right\rangle, \quad 0 \leq t \leq \tau \wedge \zeta, \quad P_{x} \text {-a.s. }
\end{aligned}
$$

Let $\left\{\tau_{k}\right\}$ be a fundamental sequence for the local martingale $\int_{0}^{\cdot \wedge \zeta}\left\langle\operatorname{sg} n\left(u(X)_{r-}\right), d M_{r}\right\rangle$. Putting $t=0$ in the above inequality with $\tau$ replaced by $\tau_{k}$ and then taking the expectation with respect to $P_{x}$ we get

$$
|u(x)| \leq E_{x}\left|u\left(X_{\tau_{k} \wedge \zeta}\right)\right|
$$

for q.e. $x \in E$. Since $u \in \mathbf{D}$, letting $k \rightarrow \infty$ we conclude that $|u|=0$ q.e.

Theorem 3.4 Assume that $\left(\mathbb{X}, \mathcal{B}_{1}, m\right)$ has the compactness property and $(\mathrm{H} 1)-(\mathrm{H} 4)$ are satisfied. Then there exists a solution of Eq. 3.1.

Proof Step 1. We first assume that $\|R|\mu|\|_{\infty}<\infty$ and there exists a strictly positive bounded Borel measurable function $g$ such that $|F(x, y)| \leq g(x)$ for $x \in E, y \in \mathbb{R}^{N}$ and $\|R g\|_{\infty}<\infty$. Let $\rho$ be a strictly positive Borel measurable function on $E$ such that $\int \rho(x) m(d x)<\infty$ and let

$$
\Phi: L_{2}(E ; \rho \cdot m) \rightarrow L_{2}(E ; \rho \cdot m), \quad \Phi(u)=R F(\cdot, u)+R \mu .
$$

The mapping $\Phi$ is well defined since $|R(F(\cdot, u))+R \mu| \leq R g+R|\mu| \in L^{2}(E ; \rho \cdot m)$. By (H3), $\Phi$ is continuous. We shall show that $\Phi$ is compact. To see this, let us consider $\left\{u_{n}\right\} \subset L^{2}(E ; \rho \cdot m)$. By Remark 3.2, the function $v_{n}=\Phi\left(u_{n}\right)$ is a probabilistic solution of the system

$$
-A v_{n}=F\left(x, u_{n}\right)+\mu .
$$


Therefore there is a MAF $M^{n}$ of $\mathbb{X}$ such that

$$
\begin{aligned}
v_{n}\left(X_{t}\right)= & v_{n}\left(X_{T \wedge \zeta}\right)+\int_{t}^{T \wedge \zeta} F\left(X_{r}, u_{n}\left(X_{r}\right)\right) d r+\int_{t}^{T \wedge \zeta} d A_{r}^{\mu} \\
& -\int_{t}^{T \wedge \zeta} d M_{r}^{n}, \quad t \in[0, \zeta \wedge T], \quad P_{x} \text {-a.s. }
\end{aligned}
$$

for q.e. $x \in E$. Hence

$$
\left|v_{n}(x)-p_{t} v_{n}(x)\right|=\left|v_{n}(x)-E_{x} v_{n}\left(X_{t \wedge \zeta}\right)\right| \leq E_{x} \int_{0}^{t \wedge \zeta} g\left(X_{r}\right) d r+E_{x} \int_{0}^{t \wedge \zeta} d A_{r}^{|\mu|}
$$

for q.e. $x \in E$. Consequently,

$$
\lim _{t \rightarrow 0^{+}} \sup _{n}\left|p_{t} v_{n}(x)-v_{n}(x)\right|=0
$$

for q.e. $x \in E$. Observe that $\left\|v_{n}\right\|_{\infty} \leq\|R g\|_{\infty}+\|R|\mu|\|_{\infty}$. Since $\left(\mathbb{X}, \mathcal{B}_{1}, m\right)$ has the compactness property, it follows from Corollary 2.7 that there is a subsequence $\left(n^{\prime}\right) \subset(n)$ such that $\left\{v_{n^{\prime}}\right\}$ converges q.e. Since $\left\{v_{n^{\prime}}\right\}$ are uniformly bounded by $\|R g\|_{\infty}+\|R \mu\|_{\infty}$, applying the Lebesgue dominated convergence theorem shows that $\left\{v_{n^{\prime}}\right\}$ converges in $L^{2}(E ; \rho \cdot m)$. By Schauder's fixed point theorem, there is $u \in L^{2}(E ; \rho \cdot m)$ such that $\Phi(u)=u$, i.e.

$$
u(x)=E_{x} \int_{0}^{\zeta} F\left(X_{r}, u\left(X_{r}\right)\right) d r+E_{x} \int_{0}^{\zeta} d A_{r}^{\mu}
$$

for $m$-a.e. $x \in E$. Let $v(x)$ be equal to the right-hand side of the above equality for $x \in E$ such that $\operatorname{Rg}(x)+R|\mu|(x)<\infty$ and zero otherwise. Then by [28, Lemma 4.2], $v$ is quasi-continuous and $v \in \mathbf{D}$. Since $v=u, m$-a.e., we have

$$
E_{x} \int_{0}^{\zeta} F\left(X_{r}, u\left(X_{r}\right)\right) d r=E_{x} \int_{0}^{\zeta} F\left(X_{r}, v\left(X_{r}\right)\right) d r
$$

for q.e. $x \in E$. Thus $v$ is a solution of Eq. 3.1 (see Remark 3.2).

Step 2. Now we consider the general case. Let $g$ be a strictly positive bounded Borel measurable function on $E$ such that $\|R g\|_{\infty}<\infty$ (for the existence of $g$ see [34, Corollary 1.3.6]) and let $\left\{F_{n}\right\}$ be a generalized nest such that $\left\|R\left|\mu_{n}\right|\right\|_{\infty}$, where $\mu_{n}=\mathbf{1}_{F_{n}} \cdot \mu$. Put

$$
F_{n}(x, y)=\frac{n g(x)}{1+n g(x)} \cdot \frac{n \cdot F(x, y)}{|F(x, y)| \vee n}, \quad x \in E, y \in \mathbb{R}^{N} .
$$

Then $F_{n}$ satisfies (H2)-(H4) and $R\left|F_{n}\right| \leq n^{2} R g$, which implies that $\left\|R\left|F_{n}\right|\right\|_{\infty}<\infty$. By Step 1 , for each $n \geq 1$ there exists a solution $u_{n}$ of the system

$$
-A u_{n}=F_{n}\left(x, u_{n}\right)+\mu_{n} .
$$

Therefore there is a MAF $M$ of $\mathbb{X}$ such that

$$
\begin{aligned}
u_{n}\left(X_{t}\right)= & u_{n}\left(X_{T \wedge \zeta}\right)+\int_{t}^{T \wedge \zeta} F_{n}\left(X_{r}, u_{n}\left(X_{r}\right)\right) d r+\int_{t}^{T \wedge \zeta} d A_{r}^{\mu_{n}} \\
& -\int_{t}^{T \wedge \zeta} d M_{r}^{n}, \quad t \in[0, T \wedge \zeta], \quad P_{x} \text {-a.s. }
\end{aligned}
$$


for q.e. $x \in E$. By [9, Theorem 3],

$$
\begin{aligned}
\left|u_{n}\left(X_{t}\right)\right| \leq & \left|u_{n}\left(X_{T \wedge \zeta}\right)\right|-\int_{t}^{T \wedge \zeta}\left\langle\operatorname{sgn}\left(u_{n}(X)_{r-}\right), F_{n}\left(X_{r}, u_{n}\left(X_{r}\right)\right)\right\rangle d r \\
& +\int_{t}^{T \wedge \zeta}\left\langle\operatorname{sg} \hat{n}\left(u_{n}(X)_{r-}\right), d A_{r}^{\mu_{n}}\right\rangle-\int_{t}^{T \wedge \zeta}\left\langle\operatorname{sg} n\left(u_{n}(X)_{r-}\right), d M_{r}^{n}\right\rangle, \quad t \in[0, T \wedge \zeta] .
\end{aligned}
$$

By the above inequality and (H4),

$$
\left|u_{n}(x)\right| \leq E_{x}\left|u_{n}\left(X_{T \wedge \zeta}\right)\right|+E_{x} \int_{0}^{T \wedge \zeta} G\left(X_{r}\right) d r+E_{x} \int_{0}^{T \wedge \zeta} d A_{r}^{|\mu|}
$$

for q.e. $x \in E$. Letting $T \rightarrow \infty$ and using the fact that $u_{n} \in \mathbf{D}$ we conclude that for q.e. $x \in E$,

$$
\left|u_{n}(x)\right| \leq E_{x} \int_{0}^{\zeta} G\left(X_{r}\right) d r+E_{x} \int_{0}^{\zeta} d A_{r}^{|\mu|} .
$$

Put $v(x)=E_{x} \int_{0}^{\zeta} G\left(X_{r}\right) d r+E_{x} \int_{0}^{\zeta} d A_{r}^{|\mu|}$ if the right-hand side of Eq. 3.6 is finite and $v(x)=0$ otherwise. By [28], $v$ is quasi-continuous, $v \in \mathbf{D}$ and $v$ is a probabilistic solution of the equation

$$
-A v=G+|\mu| .
$$

Let $U_{k}=\{v<k\}$. Since $v$ is quasi-continuous, $U_{k}$ is finely open. Moreover, since by (H1) and (H4) $v$ is finite, $\bigcup_{k=1}^{\infty} U_{k}=E$ q.e. Write $\tau_{k}=\tau_{U_{k}}$. Then

$$
\left|u_{n} \mathbf{1}_{U_{k}}(x)\right| \leq k, \quad n \geq 1, \quad x \in U_{k} .
$$

By $(\mathrm{H} 2)$,

$$
P_{x}\left(\int_{0}^{T \wedge \zeta} \sup _{|y| \leq k}|F|\left(X_{r}, y\right) d r<\infty, T>0\right)=1
$$

for every $k \geq 0$. Let

$$
\sigma_{k}=\inf \left\{t>0 ; \quad \int_{0}^{t} \sup _{|y| \leq k}|F|\left(X_{r}, y\right) d r>k\right\} .
$$

By Eq. 3.9, $\sigma_{k} \nearrow \infty$. Let $\delta_{k, l}=\tau_{k} \wedge \sigma_{l}$. By Eqs. 3.5, 3.8, 3.9 and the construction of $\delta_{k, l}$ we have

$$
\begin{aligned}
\left|u_{n}(x)-E_{x} u_{n}\left(X_{t \wedge \delta_{k, l} \wedge \zeta}\right)\right| & \leq E_{x} \int_{0}^{t \wedge \delta_{k, l} \wedge \zeta}\left|F_{n}\right|\left(X_{r}, u_{n}\left(X_{r}\right)\right) d r+E_{x} \int_{0}^{t \wedge \delta_{k, l} \wedge \zeta} d A_{r}^{|\mu|} \\
& \leq k E_{x}\left(t \wedge \delta_{k, l} \wedge \zeta\right)+E_{x} \int_{0}^{t \wedge \delta_{k, l} \wedge \zeta} d A_{r}^{|\mu|} .
\end{aligned}
$$

Hence

$$
\lim _{t \rightarrow 0^{+}} \sup _{n}\left|u_{n}(x)-E_{x} u_{n}\left(X_{t \wedge \delta_{k, l} \wedge \zeta}\right)\right|=0
$$

for q.e. $x \in E$. Now we will show that Eq. 3.10 holds for $x \in U_{k}$ with $E_{x}\left|u_{n}\left(X_{t \wedge \delta_{k, l} \wedge \zeta}\right)\right|$ replaced by $E_{x}\left[\left|u_{n}\left(X_{t \wedge \tau_{k}}\right)\right| \mathbf{1}_{\left\{t<\tau_{k}\right\}}\right]$. To this end, let us first observe that $P_{x}\left(\tau_{k}>0\right)=1$ for $x \in U_{k}$, because $U_{k}$ is finely open. We have

$$
\begin{aligned}
\sup _{n} \mid & E_{x} u_{n}\left(X_{t \wedge \delta_{k, l}}\right)-E_{x} u_{n}\left(X_{t}\right) \mathbf{1}_{\left\{t<\tau_{k}\right\}}\left|\leq E_{x} \sup _{n}\right| u_{n}\left(X_{t \wedge \delta_{k, l}}\right)-u_{n}\left(X_{t}\right) \mathbf{1}_{\left\{t<\tau_{k}\right\}} \mid \\
& =\int_{\left\{t \geq \tau_{k}\right\} \cup\left\{t \geq \delta_{k, l}\right\}} \sup _{n}\left|u_{n}\left(X_{t \wedge \delta_{k, l}}\right)-u_{n}\left(X_{t}\right) \mathbf{1}_{\left\{t<\tau_{k}\right\}}\right| d P_{x} \\
& \leq \int_{\left\{t \geq \tau_{k}\right\} \cup\left\{t \geq \delta_{k, l}\right\}}\left|v\left(X_{t \wedge \delta_{k, l}}\right)\right|+\left|v\left(X_{t}\right) \mathbf{1}_{\left\{t<\tau_{k}\right\}}\right| d P_{x} .
\end{aligned}
$$


Since $\lim _{t \rightarrow 0^{+}} \lim _{l \rightarrow \infty} P_{x}\left(\left\{t \geq \tau_{k}\right\} \cup\left\{t \geq \delta_{k, l}\right\}\right)=0$ for $x \in U_{k}$ and $v \in \mathbf{D}$, it follows that for $x \in U_{k}$ the right-hand side of the above inequality tends to zero as $l \rightarrow+\infty$ and then $t \rightarrow 0^{+}$. This and Eq. 3.10 imply that

$$
\lim _{t \rightarrow 0^{+}} \sup _{n}\left|u_{n}(x)-p_{t}^{k} u_{n}(x)\right|=0, \quad x \in U_{k},
$$

where $\left\{p_{t}^{k}, t \geq 0\right\}$ is the semigroup associated with the process $\mathbb{X}^{U_{k}}$. By Proposition 2.8 the triple $\left(\mathbb{X}^{U_{k}}, \mathcal{B}_{1}\left(U_{k}\right), m\right)$ has the compactness property. Moreover, $\mathbb{X}^{U_{k}}$ is normal since $U_{k}$ is finely open. Therefore it follows from Theorem 2.2 and Eq. 3.8 that there exists a subsequence $\left(n^{\prime}\right) \subset(n)$ such that $\left\{u_{n^{\prime}} \mathbf{1}_{U_{k}}\right\}$ is convergent q.e. By using standard argument and the fact that $\bigcup_{k} U_{k}=E$ q.e. one can now construct a subsequence $(m) \subset(n)$ such that $\left\{u_{m}\right\}$ is convergent q.e. on $E$. Without loss of generality we may assume that $(m)=(n)$. Let us write $u=\lim \sup u_{n}$ and $\delta_{k}=\delta_{k, k}$. By Eq. 3.5,

$$
\begin{aligned}
u_{n}\left(X_{t \wedge \delta_{k}}\right)= & E_{x}\left(u_{n}\left(X_{T \wedge \delta_{k}}\right)+\int_{t \wedge \delta_{k} \wedge \zeta}^{T \wedge \delta_{k} \wedge \zeta} F_{n}\left(X_{r}, u_{n}\left(X_{r}\right)\right) d r\right. \\
& \left.+\int_{t \wedge \delta_{k} \wedge \zeta}^{T \wedge \delta_{k} \wedge \zeta} d A_{r}^{\mu_{n}} \mid \mathcal{F}_{t \wedge \delta_{k} \wedge \zeta}\right), \quad t \in[0, T], \quad P_{x} \text {-a.s., }
\end{aligned}
$$

so applying [10, Lemma 6.1] we can conclude that for every $q \in(0,1)$,

$$
\begin{aligned}
& E_{x} \sup _{t \leq \delta_{k} \wedge T}\left|u_{n}\left(X_{t}\right)-u_{m}\left(X_{t}\right)\right|^{q} \leq \frac{1}{1-q}\left[E_{x}\left|u_{n}\left(X_{\delta_{k} \wedge T}\right)-u_{m}\left(X_{\delta_{k} \wedge T}\right)\right|\right. \\
& \left.\quad+E_{x} \int_{0}^{T \wedge \delta_{k} \wedge \zeta}\left|F_{n}\left(X_{r}, u_{n}\left(X_{r}\right)\right)-F_{m}\left(X_{r}, u_{m}\left(X_{r}\right)\right)\right| d r+E_{x} \int_{0}^{T \wedge \delta_{k} \wedge \zeta} d A_{r}^{\left|\mu_{n}-\mu_{m}\right|}\right]^{q} .
\end{aligned}
$$

Applying the Lebesgue dominated convergence theorem and using (H3), the construction of $F_{n},\left\{\delta_{k}\right\}$ and the convergence of $\left\{u_{n}\right\}$ we conclude that for q.e. $x \in E$ the first and second term on the right-hand side of the above inequality converges to zero as $n, m \rightarrow \infty$. To show the convergence of the third term, let us observe that

$$
A_{t}^{\left|\mu_{n}-\mu_{m}\right|}=\int_{0}^{t} \mathbf{1}_{F_{n} \Delta F_{m}}\left(X_{r}\right) d A_{r}^{\mu}, \quad t \geq 0 .
$$

Since $E_{x} \int_{0}^{\zeta} d A_{r}^{|\mu|}<\infty$ q.e., it is enough to show that

$$
\lim _{n, m \rightarrow \infty} P_{x}\left(\exists_{t>0} X_{t} \in F_{n} \Delta F_{m}\right)=\lim _{n, m \rightarrow \infty} P_{x}\left(\sigma_{F_{n} \Delta F_{m}}<\infty\right)=0
$$

for q.e. $x \in E$. But this follows immediately from the fact that $\left\{F_{n}\right\}$ is a nest (see [34, Theorem 3.4.8]). By what has already been proved,

$$
\left(u_{n}(X), \int_{0}^{\cdot} F_{n}\left(X_{r}, u_{n}\left(X_{r}\right)\right) d r, A^{\mu_{n}}\right) \rightarrow\left(u(X), \int_{0}^{\cdot} F\left(X_{r}, u\left(X_{r}\right)\right) d r, A^{\mu}\right),
$$

uniformly on compacts in probability $P_{x}$ for q.e. $x \in E$. Therefore letting $n \rightarrow \infty$ in Eq. 3.5 we see that there exists a local MAF $M$ of $\mathbb{X}$ such that Eq. 3.2 is satisfied for q.e. $x \in E$. The fact that $u \in \mathbf{D}$ and $u$ satisfies condition (b) of the definition of a probabilistic solution of Eq. 3.1 follows from Eqs. 3.6 and 3.7.

\section{Systems with Operators Generated by Right Markov Processes}

In the present section we assume that $\mathbb{X}$ is a general transient right Markov process on $E$ satisfying hypothesis (L) of Meyer. 
Let us fix an excessive ( $\sigma$-finite) measure $m$ on $E$, i.e. a Borel measure on $\mathcal{B}(E)$ such that

$$
m \circ \alpha R_{\alpha} \leq m,
$$

where $\left(m \circ \alpha R_{\alpha}\right) f=m\left(\alpha R_{\alpha} f\right)=\int f(x) m(d x)$ for $f \in \mathcal{B}^{+}(E)$.

We say that a set $B \subset E$ is $m$-polar if there exists an excessive function $v$ such that $A \subset\{v=\infty\}$ and $v$ is finite $m$-a.e.

In this section we say that a property holds q.e. if it holds except for some $m$-polar set.

Recall that a set $N \in \mathcal{B}^{n}(E)$ is $m$-inessential if it is $m$-polar and absorbing for $\mathbb{X}$.

Definition An $\mathcal{F}$-adapted increasing $[0, \infty]$-valued process $\left\{A_{t}, t \geq 0\right\}$ is called positive co-natural additive functional (PcNAF) of $\mathbb{X}$ if there exist a defining set $\Omega_{A} \subset \mathcal{F}_{\infty}$ and an $m$-inessential Borel set $N_{A} \subset E$ such that

(a) $P_{x}\left(\Omega_{A}\right)=1$ for $x \notin N_{A}$ and $\theta_{t} \Omega_{A} \subset \Omega_{A}, t \geq 0$,

(b) for every $\omega \in \Omega_{A}$ the mapping $t \mapsto A_{t}(\omega)$ is right continuous on $[0, \infty)$ and finite valued on $[0, \zeta)$ with $A_{0}(\omega)=0$,

(c) for every $\omega \in \Omega_{A}$ and $t>0, \Delta A_{t} \equiv A_{t}-A_{t-}=a\left(X_{t}\right)$, where $a \in p \mathcal{B}^{n}(E)$,

(d) for every $w \in \Omega_{A}, A_{t+s}(\omega)=A_{t}(\omega)+A_{s}\left(\theta_{t} \omega\right)$ for all $s, t \geq 0$.

Remark 4.1 It is known (see [24, Proposition 6.12]) that for any $m$-polar set $N$ there exists a Borel $m$-inessential set $B$ such that $N \subset B$. Therefore if some property holds q.e. then without loss of generality we may assume that it holds everywhere except for possibly an $m$-inessential set.

Given a PcNAF $A$ and $f \in \mathcal{B}^{+}(E)$ set

$$
U_{A} f(x)=E_{x} \int_{0}^{\zeta} f\left(X_{r}\right) d A_{r}, \quad x \in E .
$$

By $\mu_{A}$ we denote the Revuz measure associated with $A$, i.e. the measure defined as

$$
\mu_{A}(f)=\sup \left\{v \circ U_{A} f ; \quad v \circ U \leq m\right\} .
$$

In this section by a nest we understand an increasing sequence $\left\{B_{n}\right\}$ of nearly Borel sets such that $P_{m}\left(\lim _{n \rightarrow \infty} \tau_{B_{n}}<\zeta\right)=0$.

Definition A Borel measure $\mu$ on $E$ is called smooth if it charges no $m$-polar sets and there exists a nest $\left\{G_{n}\right\}$ of finely open nearly Borel sets such that $\mu\left(G_{n}\right)<\infty, n \geq 1$.

It is known (see [19, Therems 6.15, 6.21, 6.29]) that for every PcNAF $A$ its Revuz measure $\mu_{A}$ is smooth and for every smooth measure $\mu$ there exists a unique PcNAF $A^{\mu}$ such that its Revuz measure is equal to $\mu$.

Proposition $4.2\left(\mathbb{X}, \mathcal{B}_{1}, m\right)$ has the compactness property iff $(\mathbb{X},[0, v], m)$ has the compactness property for every $v \in \mathbf{D}$.

Proof Sufficiency is obvious. To prove necessity, let us assume that $\left(\mathbb{X}, \mathcal{B}_{1}, m\right)$ has the compactness property and for $v \in \mathbf{D}$ let us choose $\left\{u_{n}\right\} \subset \mathcal{B}^{+}(E)$ such that $u_{n} \leq v, m$ a.e. for $n \geq 1$. Write $u_{n}^{k}=T_{k}\left(u_{n}\right)$. Since $v \in \mathbf{D}, R_{\alpha} v$ is finite $m$-a.e. for every $\alpha>$ 0 . Let $g$ be a strictly positive Borel measurable function on $E$ such that $\int\left(R_{\alpha} v\right) g d m<$ $\infty$. By the assumption, for every $k \geq 0$ there exists a subsequence $\left(n^{\prime}\right) \subset(n)$ such that 
$\left\{R_{\alpha} u_{n^{\prime}}^{k}\right\}$ is convergent in $L^{1}(E ; g \cdot m)$. Therefore to show the existence of a subsequence (m) $\subset$ (n) such that $R_{\alpha} u_{m}$ converges in $L^{1}(E ; g \cdot m)$ it is enough to prove that $\| R_{\alpha} u_{n}^{k}-$ $R_{\alpha} u_{n} \|_{L^{1}(E ; g \cdot m)} \leq C(k)$ for some independent of $n$ constants $C(k)$ such that $C(k) \rightarrow 0$ as $k \rightarrow+\infty$. Observe that

$$
\begin{aligned}
\left\|R_{\alpha} u_{n}^{k}-R_{\alpha} u_{n}\right\|_{L^{1}(E ; g \cdot m)} & \leq E_{g \cdot m} \int_{0}^{\infty} e^{-\alpha t}\left|u_{n}\left(X_{t}\right)-u_{n}^{k}\left(X_{t}\right)\right| d t \\
& \leq E_{g \cdot m} \int_{0}^{\infty} e^{-\alpha t} \mathbf{1}_{\left\{v\left(X_{t}\right)>k\right\}} v\left(X_{t}\right) d t \equiv C(k) .
\end{aligned}
$$

Since $\int\left(R_{\alpha} v\right) g d m<\infty, C(k) \rightarrow 0$ as $k \rightarrow \infty$.

Proposition 4.3 Let $\mathbb{X}$ be a right Markov process and $m$ be an excessive measure. Then $\left(\mathbb{X}, \mathcal{B}_{1}, m\right)$ has the compactness property iff $R_{\alpha}: L^{1}(E ; m) \rightarrow L^{1}(E ; m)$ is order compact for some (and hence for every) $\alpha>0$.

Proof Necessity. Assume that $\left(\mathbb{X}, \mathcal{B}_{1}(E), m\right)$ has the compactness property. Let $v \in$ $L^{1}(E ; m)$ and $\left\{u_{n}\right\} \subset[0, v]$. Write $v_{n}^{k}=R_{\alpha} u_{n}^{k}, v_{n}=R_{\alpha} u_{n}, u_{n}^{k}=T_{k}\left(u_{n}\right)$ for $n, k \geq 1$. By the compactness property of $\left(\mathbb{X}, \mathcal{B}_{1}(E), m\right)$ there exists a subsequence (still denoted by $(n))$ such that $v_{n}^{k}$ is $m$-a.e. convergent. Since $v_{n}^{k} \leq R_{\alpha} v \in L^{1}(E ; m), v_{n}^{k}$ is convergent in $L^{1}(E ; m)$. Furthermore,

$$
\begin{aligned}
\left\|v_{n}^{k}-v_{n}\right\|_{L^{1}(E ; m)} \leq \int_{E} R_{\alpha}\left|T_{k}\left(u_{n}\right)-u_{n}\right| d m & \leq \frac{1}{\alpha} \int_{E}\left|T_{k}\left(u_{n}\right)-u_{n}\right| d m \\
& \leq \frac{2}{\alpha} \int_{\{v>k\}} v d m \equiv C(k) .
\end{aligned}
$$

Since $C(k) \rightarrow 0$ as $k \rightarrow+\infty$, there exists a subsequence $\left(n^{\prime}\right) \subset(n)$ such that $\left\{v_{n^{\prime}}\right\}$ is convergent in $L^{1}(E ; m)$.

Sufficiency. Now assume that $R_{\alpha}: L^{1}(E ; m) \rightarrow L^{1}(E ; m)$ is order compact. Let $\left\{u_{n}\right\} \subset$ $\mathcal{B}^{+}(E)$ be such that $u_{n}(x) \leq 1$ for $x \in E, n \geq 1$. Let $\left\{g_{k}\right\}$ be a sequence of positive functions in $L^{1}(E ; m)$ such that $g_{k} \nearrow 1$ and let $\rho$ be a strictly positive function in $L^{1}(E ; m)$. Write $u_{n}^{k}=u_{n} g_{k}, v_{n}^{k}=R_{\alpha} u_{n}^{k}, v_{n}=R_{\alpha} u_{n}$. By the assumption, for every $k \geq 1$ there exists a subsequence (still denoted by $n$ ) such that $v_{n}^{k}$ converges in $L^{1}(E ; m)$. It follows that for every $k \geq 1$ there exists a subsequence (still denoted by $n$ ) such that $v_{n}^{k}$ converges in $L^{1}(E ; \rho \cdot m)$. This when combined with the fact that

$$
\lim _{k \rightarrow \infty}\left\|v_{n}^{k}-v_{n}\right\|_{L^{1}(E ; \rho \cdot m)} \leq \lim _{k \rightarrow \infty} \int_{E} R_{\alpha}\left|1-g_{k}\right| \rho d m=0
$$

implies the existence of a subsequence $\left(n^{\prime}\right) \subset(n)$ such that $v_{n^{\prime}}$ converges in $L^{1}(E ; \rho \cdot m)$. Therefore there is a further subsequence $\left(n^{\prime \prime}\right) \subset\left(n^{\prime}\right)$ such that $v_{n^{\prime \prime}}$ converges $m$-a.e.

Let us consider the following system

$$
-A u=F(x, u)+\mu,
$$

where $(A,(D(A))$ is the operator defined by

$$
D(A)=R_{\alpha}\left(L^{1}(E ; m)\right), \quad-A\left(R_{\alpha} f\right)=f-\alpha R_{\alpha} f, \quad f \in L^{1}(E ; m) .
$$

for some $\alpha>0$. Since $m$ is an excessive measure,

$$
\int_{E} \alpha R_{\alpha} f d m \leq \int_{E} f d m, \quad f \in \mathcal{B}^{+}(E)
$$


from which it follows immediately that $R_{\alpha} f=0, m$-a.e., if $f=0, m$-a.e. Therefore Eq. 4.2 makes sense. Also note that by the resolvent equation the definition of $(A, D(A))$ is independent of $\alpha>0$.

Proposition 4.4 Let $B \in \mathcal{B}(E)$. If ( $\mathbb{X}, \mathcal{B}_{1}(E)$ ) has the compactness property then $\left(\mathbb{X}^{B}, \mathcal{B}_{1}(E)\right)$ has the compactness property.

Proof Follows by the same method as in the proof of Proposition 2.8, because under the assumption of the present proposition we need not use Lemma 2.6.

Theorem 4.5 Let $\mathbb{X}$ be a transient Markov process satisfying condition (L) of Meyer. Assume that (H1)-(H4) are satisfied. Then there exists a solution of Eq. 4.1).

Proof We assume that there exists a Borel function $g \in \mathcal{B}^{+}(E)$ such that $|F(x, y)| \leq g(x)$ for $x \in E, y \in \mathbb{R}^{N}$ and $R g$ is finite $m$-a.e. Let $\rho$ be a strictly positive Borel function on $E$ such that

$$
\int_{E}\left[E_{x} A_{\zeta}^{|\mu|}+R g(x)\right]^{2} \rho(x) m(d x)<\infty
$$

Let

$$
\Phi: L_{2}(E ; \rho \cdot m) \rightarrow L_{2}(E ; \rho \cdot m), \quad \Phi(u)=R F(\cdot, u)+R \mu .
$$

The mapping $\Phi$ is well defined since

$$
|R(F(\cdot, u))+R \mu| \leq R g \in R|\mu| \in L^{2}(E ; \rho \cdot m) .
$$

In fact $\Phi: L^{2}(E ; \rho \cdot m) \rightarrow B_{L^{2}(E ; \rho \cdot m)}(0, r)$, where $r=\|R g\|_{L^{2}(E ; \rho \cdot m)}+\|R|\mu|\|_{L^{2}(E ; \rho \cdot m)}$. $\Phi$ is continuous by (H3). Let $\left\{u_{n}\right\} \subset L^{2}(E ; \rho \cdot m)$. Define $v_{n}$ by putting $v_{n}(x)=$ $R F\left(\cdot, u_{n}\right)(x)+R \mu(x)$ for $x$ such that $R g(x)+R|\mu|(x)<\infty$ and $v_{n}(x)=0$ otherwise. By the assumptions, [39, Theorems 36.10, 49.9] and the definition of $m$-polar sets $v_{n}$ is finely continuous and finite q.e. By Remark 4.1 we may assume that it is finite except for an $m$-inessential set. Then by the strong Markov property formula (3.4) holds. Therefore repeating the arguments following (3.4) and applying Proposition 4.2 we conclude that $\Phi$ is compact. The rest of the proof now runs as in Step 2 of the proof of Theorem 3.4 (we use Proposition 4.4 instead of Proposition 2.8).

\section{Applications}

In this section we give several examples of processes having the compactness property.

Example 5.1 Let $\left\{\mu_{t}, t>0\right\}$ be a convolution semigroup on $\mathbb{R}^{d}$ and let $\mathbb{X}$ be a Hunt process with the transition function

$$
p_{t} f(x)=\int_{\mathbb{R}^{d}} f(x+y) \mu_{t}(d y) .
$$

It is known (see [5]) that if for some $\varepsilon>0$

$$
\lim _{|x| \rightarrow \infty}|x|^{-\varepsilon}|\operatorname{Re} \psi|(x) \rightarrow \infty,
$$


where $\hat{\mu}_{t}(x)=e^{-t \psi(x)}, x \in \mathbb{R}^{d}\left(\hat{\mu}_{t}\right.$ stands for the Fourier transform of $\left.\mu_{t}\right)$ then the Lebesgue measure $m$ on $\mathbb{R}^{d}$ is a reference measure for $\mathbb{X}$. Therefore if $\mathbb{X}$ is a Lévy process with the characteristic exponent $\psi$ satisfying (5.1) then $\left(\mathbb{X}, \mathcal{B}_{1}\right)$ has the compactness property. Consequently, our existence and uniqueness results of Section 3 (Theorem 3.4 and Proposition 3.3) apply to systems with operator $A$ of the form $\psi(\nabla)$ with $\psi$ satisfying (5.1). A model example is $\psi$ of the form $\psi(x)=|x|^{\alpha}, x \in \mathbb{R}^{d}$, for some $\alpha \in(0,2]$, which corresponds to the fractional Laplacian $\psi(\nabla)=\left(\nabla^{2}\right)^{\alpha / 2}=\Delta^{\alpha / 2}$.

Example 5.2 Let $H$ be a real Hilbert space, $Q \in \mathcal{L}(H)$ be a selfadjoint nonnegative operator and $A$ be a generator of a $C_{0}$-semigroup $e^{t A}$ on $H$. Let

$$
Q_{t}=\int_{0}^{t} e^{s A} Q e^{s A^{*}} d s
$$

be of trace class, $e^{t A}(H) \subset Q_{t}^{1 / 2}(H)$ and $\operatorname{Ker} Q_{t}=\{0\}, t>0$. It is well known that the Ornstein-Uhlenbeck semigroup

$$
\left(T_{t} \phi\right)(x)=\int_{H} \phi(y) \mathcal{N}\left(e^{t A} x, Q_{t}\right)(d y), \quad \phi \in \mathcal{B}_{b}(H),
$$

where $\mathcal{N}\left(e^{t A} x, Q_{t}\right)$ is the Gaussian measure on $H$ with mean $e^{t A} x$ and covariance operator $Q_{t}$ is representable by the Ornstein-Uhlenbeck process being a solution of the SDE

$$
\left\{\begin{array}{l}
d X(t, x)=A X(t, x) d t+Q^{1 / 2} d W(t) \\
X(0, x)=x \in H
\end{array}\right.
$$

i.e.

$$
\left(T_{t} \phi\right)(x)=E_{x} \phi\left(X_{t}\right), \quad \phi \in \mathcal{B}_{b}(H)
$$

(see [15] for details). By the Cameron-Martin formula (see, e.g., [14]), for every $x \in H$ the measure $\mathcal{N}\left(e^{t A} x, Q_{t}\right)$ is equivalent to the measure $\mathcal{N}\left(0, Q_{t}\right)$. Therefore $\mathbb{X}$ satisfies Meyer's hypothesis $(\mathrm{L})$, which implies that $\left(\mathbb{X}, \mathcal{B}_{1}\right)$ has the compactness property. It follows that the results of Section 3 apply to systems with Ornstein-Uhlenbeck operator being a generator of the semigroup $\left\{T_{t}\right\}$.

Example 5.3 Let $(\mathcal{E}, D[\mathcal{E}])$ be a regular symmetric Dirichlet form on $L^{2}(E ; m)$. By [23], if the following Sobolev type inequality holds

$$
\|u\|_{p_{0}}^{2} \leq c \mathcal{E}_{\lambda_{0}}(u, u), \quad u \in D[\mathcal{E}]
$$

for some $c>0, p_{0}>2, \lambda_{0} \geq 0$, then $m$ is a reference measure for $\mathbb{X}$ associated with $(\mathcal{E}, D[\mathcal{E}])$. Consequently, $\left(\mathbb{X}, \mathcal{B}_{1}\right)$ has the compactness property.

Example 5.4 Let $(\mathcal{E}, D[\mathcal{E}])$ be a regular semi-Dirichlet form and let $\mathbb{X}$ be the associated Hunt process. Suppose that $(\mathbb{X}, \mathcal{P}, m)$ has the compactness property. Let $\mu$ be a positive smooth measure and let $\left(\mathcal{E}_{\mu}, D\left[\mathcal{E}_{\mu}\right]\right)$ be the form defined as

$$
\mathcal{E}_{\mu}(u, v)=\mathcal{E}(u, v)+\int_{E} u v d \mu, \quad D\left[\mathcal{E}_{\mu}\right]=\left\{u \in D[\mathcal{E}] ; \int_{E}|u|^{2} d \mu<\infty\right\} .
$$

It is known that $\left(\mathcal{E}_{\mu}, D\left[\mathcal{E}_{\mu}\right]\right)$ is a quasi-regular Dirichlet form and that the associated standard special process $\mathbb{X}^{\mu}$ is a subprocess of $\mathbb{X}$ (see [23, Section 6.4]). Therefore $\left(\mathbb{X}{ }^{\mu}, \mathcal{P}, m\right)$ has the compactness property.

Example 5.5 Let $\left(\left\{X_{t}, t \geq 0\right\},\left\{P_{s, x},(s, x) \in[0, \infty) \times E\right\}\right)$ be a time inhomogenous Markov process. Assume that for every $s \geq 0$ the pair $\left(X^{s}=\left(\left\{X_{s+t}, t \geq 0\right\},\left\{P_{s, x}, x \in\right.\right.\right.$ 
$\left.E\}), \mathcal{B}_{1}(E)\right)$ has the compactness property. Then by Proposition $2.4, X^{s}$ has a reference measure $m(s)$. Assume that $m(s)=m, s \geq 0$. Let $\mathbf{Z}=\left(\left\{Z_{t}, t \geq 0\right\},\left\{P_{z}, z \in[0, \infty) \times E\right\}\right)$, where $Z_{t}=\left(\tau(t), X_{\tau(t)}\right), t \geq 0$ and $\tau$ is the uniform motion to the right i.e. $\tau(t)=\tau(0)+t$, $P_{s, x}(\tau(0)=s)=1$. Then $\mathbf{Z}$ is a Markov process with reference measure $\bar{m}=d t \otimes m$. Indeed, we have

$$
R_{\alpha}^{\mathbf{Z}}((s, x), T \times B)=\int_{0}^{\infty} e^{-\alpha t} E_{s, x} \mathbf{1}_{B}\left(X_{s+t}\right) \cdot \mathbf{1}_{T}(s+t) d t .
$$

Suppose that $T \in \mathcal{B}([0, \infty)), B \subset \mathcal{B}(E)$ and $\bar{m}(T \times B)=0$. Then $d t(T)=0$ or $m(B)=0$. If $d t(T)=0$ then it is clear that $R_{\alpha}^{\mathbf{Z}}((s, x), T \times B)=0$. If $m(B)=0$ then the right-hand side of Eq. 5.2 is less then or equal to

$$
\int_{0}^{\infty} e^{-\alpha t} E_{s, x} \mathbf{1}_{B}\left(X_{s+t}\right) d t=R_{\alpha}^{\mathbb{X}^{s}}(x, B)=0,
$$

the last equality being a consequence of the fact that $R_{\alpha}^{\mathbb{X}^{s}}(x, d y) \ll m(d y)$. Thus $\bar{m}$ is the reference measure. As a result, the pair $\left(\mathbf{Z}, \mathcal{B}_{1}([0, \infty) \times E)\right)$ has the compactness property. For instance, let $\{A(t), t \geq 0\}$ be a family of operators associated with regular semi-Dirichlet forms $\mathcal{E}^{(t)}$ on $L^{2}(E ; m)$ and let $\mathbf{Z}$ be a process associated with the operator $\mathcal{L}=\frac{\partial}{\partial t}+A(t)$. If for every $t \geq 0$ the Hunt processes associated with $\mathcal{E}^{(t)}$ together with $\mathcal{B}_{1}(E)$ form pairs having the compactness property with the same reference measure then the pair $\left(\mathbf{Z}, \mathcal{B}_{1}([0, \infty) \times E)\right)$ has the compactness property.

Example 5.6 Let $\mathbb{X}$ be a solution of the following $d$-dimensional SDE

$$
X_{t}^{x}=x+\sum_{j=1}^{d} \int_{0}^{t} a_{j}\left(r, X_{r}^{x}\right) d W_{r}^{j}+\int_{0}^{t} b\left(r, X_{r}^{x}\right) d r,
$$

where $x \in \mathbb{R}^{d}$ and $a_{j}, b:[0, \infty] \times \mathbb{R}^{d} \rightarrow \mathbb{R}^{d}, j=1, \ldots, d$, are measurable functions satisfying the assumptions

(a) $\sum_{j=1}^{d}\left|a_{j}(t, x)-a_{j}(t, y)\right|+|b(t, x)-b(t, y)| \leq L|x-y|$ for every $x, y \in \mathbb{R}^{d}, t \geq 0$, (b) $t \rightarrow a_{j}(t, 0), t \rightarrow b(t, 0)$ are bounded on $[0, T]$ for every $T>0$.

Then by [33, Theorem 2.3.1], if

$$
P\left(S_{x}=0\right)=1,
$$

where

$$
S_{x}=\inf \left\{t>0 ; \int_{0}^{t} \mathbf{1}_{\left\{\operatorname{det} \sigma\left(r, X_{r}\right) \neq 0\right\}} d r>0\right\} \wedge T, \quad \sigma=a \cdot a^{T},
$$

then for every $t>0$ the distribution of $X_{t}$ is absolutely continuous with respect to the Lebesgue measure on $\mathbb{R}^{d}$. It follows that if, for instance, $\sigma(t, x)>0$ for every $(t, x) \in$ $[0, \infty) \times \mathbb{R}^{d}$, then $\left(\mathbb{X}, \mathcal{B}_{1}\left(\mathbb{R}^{d}\right)\right)$ has the compactness property. More generally, let $A$ be an absorbing set for $\mathbb{X}$, i.e. if $x \notin A$ then $P\left(\exists_{t} X_{t}^{x} \in A\right)=0$. Then if $\sigma(t, x)>0$ for every $(t, x) \in[0, \infty) \times \mathbb{R}^{d} \backslash A$ then $\left(\mathbb{X}^{d} \backslash A, \mathcal{B}_{1}(E \backslash A)\right)$ has the compactness property. To be more specific, let us consider diffusion process describing dividend-paying asset prices in the classical multidimentional Black and Scholes model, i.e.

$$
X_{t}^{x, i}=x_{i}+\int_{0}^{t}\left(r-d_{i}\right) X_{r}^{x, i} d r+\sum_{j=1}^{d} \int_{0}^{t} \sigma_{i j} X_{r}^{x, i} d W_{r}^{j}, \quad i=1, \ldots, d .
$$

Then $\left(\mathbb{X}^{\mathbb{R}^{d} \backslash A}, \mathcal{B}_{1}\left(\mathbb{R}^{d} \backslash A\right)\right.$ ), where $A=\left\{x \in \mathbb{R}^{d}: x_{i}=0\right.$ for some $\left.i=1, \ldots, d\right\}$, has the compactness property. 
We close this section with an example of a right Markov process $\mathbb{X}$ which is not associated with a Dirichlet form, so that the results of Section 3 can not be applied to systems with operator associated with $\mathbb{X}$. However, $\mathbb{X}$ satisfies Meyer's hypothesis (L), so that results of Section 4 are applicable.

Example 5.7 For $\phi \in C^{1}\left(\mathbb{R}^{2}\right), x \in \mathbb{R}^{2}$ set

$$
L \phi(x)=\frac{1}{2} \operatorname{tr}\left(Q D^{2} \phi(x)\right)+\langle A x, D \phi(x)\rangle,
$$

where

$$
Q=\left[\begin{array}{ll}
1 & 1 \\
1 & 1
\end{array}\right], \quad A=\left[\begin{array}{cc}
-1 & 0 \\
0 & -2
\end{array}\right]
$$

Then the semigroup $e^{t A}$ generated by $A$ is of the form

$$
e^{t A}=\left[\begin{array}{cc}
e^{t} & 0 \\
0 & e^{-2 t}
\end{array}\right], \quad t \geq 0
$$

and

$$
Q_{t} \equiv \int_{0}^{t} e^{s A} Q e^{s A} d s=\left[\begin{array}{cc}
\frac{1}{2}\left(1-e^{-2 t}\right) & \frac{1}{3}\left(1-e^{-3 t}\right) \\
\frac{1}{3}\left(1-e^{-3 t}\right) & \frac{1}{4}\left(1-e^{-4 t}\right),
\end{array}\right], \quad Q_{\infty}=\left[\begin{array}{cc}
\frac{1}{2} & \frac{1}{3} \\
\frac{1}{3} & \frac{1}{4}
\end{array}\right] .
$$

It is clear that $\operatorname{Ker} Q_{t}=\{0\}$ and $Q_{t}>0$ for every $t>0$. Let $\left\{P_{t}, t \geq 0\right\}$ be the semigroup generated by the operator $L$ on $L^{2}\left(\mathbb{R}^{2} ; \mu\right)$, where $\mu=\mathcal{N}\left(0, Q_{\infty}\right)$. It is well known that

$$
P_{t} f(x)=E_{x} f\left(X_{t}\right),
$$

where $X$ is a unique solution of the SDE

$$
d X_{t}=A X_{t} d t+Q^{1 / 2} d W_{t}, \quad X_{0}=x .
$$

From [30, 31] it follows that $\left.\mathbb{X}=\left\{\left(X, P_{x}\right), x \in \mathbb{R}^{2}\right\}\right)$ satisfies Meyer's hypthesis (L). Therefore $\left(\mathbb{X}, \mathcal{B}_{1}\right)$ has the compactness property. On the other hand, by [25], $\left\{P_{t}, t \geq 0\right\}$ is variational (i.e. is associated with a Dirichlet form on $\left.L^{2}\left(\mathbb{R}^{2}, \mu\right)\right)$ if and only if $\left\{P_{t}, t \geq 0\right\}$ is analytic. By [25] (see also [22]), $\left\{P_{t}, t \geq 0\right\}$ is analytic if and only if $Q$ is invertible. Accordingly, $\left\{P_{t}, t \geq 0\right\}$ is not variational.

Acknowledgments Research supported by Polish NCN grant no. 2012/07/D/ST1/02107.

Open Access This article is distributed under the terms of the Creative Commons Attribution 4.0 International License (http://creativecommons.org/licenses/by/4.0/), which permits unrestricted use, distribution, and reproduction in any medium, provided you give appropriate credit to the original author(s) and the source, provide a link to the Creative Commons license, and indicate if changes were made.

\section{References}

1. Alibaud, N., Andreianov, B., Bendahmane, M.: Renormalized solutions of the fractional Laplace equations. C.R. Acad. Sci. Paris, Ser. I 348, 759-762 (2010)

2. Aliprantis, C.D., Burkinshaw, O.: Positive compact operators on Banach lattices. Math. Z. 174, 289-298 (1980)

3. Bénilan, P., Brezis, H.: Nonlinear problems related to the Thomas-Fermi equation. J. Evol. Equ. 3, 673770 (2004)

4. Bénilan, P., Boccardo, L., Gallouët, T., Gariepy, R., Pierre, M., Vazquez, J.-L.: $L^{1}$-theory of existence and uniqueness of solutions of nonlinear elliptic equations. Ann. Scuola Norm. Sup. Pisa Cl. Sci. 22, 241-273 (1995) 
5. Bertoin, L.: Lévy Processes. Cambridge University Press, Cambridge (1996)

6. Beznea, L., Boboc, N.: Balayages on excessive measures, their representation and the quasi-Lindelöf property. Potential Anal. 7, 805-825 (1997)

7. Beznea, L., Boboc, N.: Potential Theory and Right Processes. Kluwer Academic Publishers, Dordrecht (2004)

8. Boboc, N., Bucur, Gh., Cornea, A.: Order and Convexity in Potential Theory: H-cones. Lecture Notes in Math, vol. 853. Springer, Berlin (1981)

9. Bouleau, N.: Formules de changement de variables. Ann. Inst. H. Poincaré Probab. Statist. 20, 133-145 (1984)

10. Briand, Ph., Delyon, B., Hu, Y., Pardoux, E., Stoica, L.: $L^{p}$ solutions of Backward Stochastic Differential Equations. Stochastic Process. Appl. 108, 109-129 (2003)

11. Blumenthal, M.R., Getoor, R.K.: Markov Processes and Potential Theory. Dover Publications, New York (2007)

12. Boccardo, L., Gallouët, T., Orsina, L.: Existence and uniqueness of entropy solutions for nonlinear elliptic equations with measure data. Ann. Inst. H. Poincaré Anal. Non Linéare 13, 539-551 (1996)

13. Boccardo, L., Dall'Aglio, A., Gallouët, T., Orsina, L.: Nonlinear Parabolic Equations with Measure Data. J. Funct. Anal. 147, 237-258 (1997)

14. Da Prato, G.: An Introduction to Infinite-Dimensional Analysis. Springer, Berlin (2006)

15. Da Prato, G., Zabczyk, J.: Stochastic Equation in Infinite Dimension. Cambridge University Press, Cambridge (1992)

16. Dal Maso, G., Murat, F., Orsina, L., Prignet, A.: Renormalized solutions of elliptic equations with general measure data. Ann. Scuola Norm. Sup. Pisa Cl. Sci. 28, 741-808 (1999)

17. Dellacherie, C., Meyer, P.A.: Probabilities and potential C. North-Holland, Amsterdam (1988)

18. Droniou, J., Porretta, A.: Prignet, A.:Parabolic Capacity and Soft Measures for Nonlinear Equations. Potential Anal. 19, 99-161 (2003)

19. Fitzsimmons, P.J., Getoor, R.K.: Homogeneous Random Measure and Strongly Supermedian Kernels of a Markov Process. Electron. J. Probab. 8, 1-54 (2003)

20. Fuglede, B.: Integral Representation of Fine Green Potentials. Math. Ann. 262, 191-214 (1983)

21. Fuglede, B.: The Dirichlet Laplacian on Finely Open Sets. Potential Anal. 10, 91-101 (1999)

22. Fuhrman, M.: Analyticity of transition semigroups and closability of bilinear forms in Hilbert space. Studia Math. 115, 53-71 (1995)

23. Fukushima, M., Oshima, Y., Takeda, M.: Dirichlet Forms and Symmetric Markov Processes. Walter de Gruyter, Berlin (1994)

24. Getoor, R.K., Sharpe, M.J.: Naturality, standardness, and weak duality for Markov processes. Z Wahrsch. verw. Gebiete 67, 1-62 (1984)

25. Goldys, B.: On analiticity of Ornstein-Uhlenbeck semigroups. Rend Mat. Acc. Lincei 9, 131-140 (1999)

26. Karlsen, K.H., Petitta, F., Ulusoy, S.: A duality approach to the fractional Laplacian with measure data. Publ. Mat. 55, 151-161 (2011)

27. Klimsiak, T.: Semilinear elliptic systems with measure data. Ann. Mat. Pura Appl. 194(4), 55-76 (2015)

28. Klimsiak, T., Rozkosz, A.: Dirichlet forms and semilinear elliptic equations with measure data. J. Funct. Anal. 265, 890-925 (2013)

29. Klimsiak, T., Rozkosz, A.: Semilinear elliptic equations with measure data and quasi-regular Dirichlet forms. arXiv: $1307.0717 \mathrm{v} 2$

30. Kuptsov, L.P.: Fundamental solutions for a class of second-order elliptic-parabolic equations. Differentcial'nye Uravnenija 8, 1649-1660 (1972)

31. Kuptsov, L.P.: English Translation. Differ. Equ. 8, 1269-1278 (1972)

32. Ma, Z., Röckner, M.: Introduction to the Theory of (Non-Symmetric) Dirichlet Forms. Springer, Berlin (1992)

33. Nualart, D.: The Malliavin calculus and related topics. Springer, New York (1995)

34. Oshima, Y.: Semi-Dirichlet Forms and Markov Processes. Walter de Gruyter, Berlin (2013)

35. Revuz, D.: Mesures associes aux fonctionelles additives de Markov. I. Trans. Amer. Math. Soc. 148, 501-531 (1970)

36. Revuz, D.: Markov Chains. 2nd Edn. North-Holland, Amsterdam (1984)

37. Sato, S.: On the reconstruction of a killed Markov process. Séminaire de probabilités XXVI. Lecture Notes Math. 1526, 540-559 (1992)

38. Serrin, J.: Pathological solutions of elliptic differential equations. Ann. Scuola Norm. Sup. Pisa 18(3), 385-387 (1964)

39. Sharpe, M.: General Theory of Markov Processes. Academic Press, New York (1988) 
40. Stampacchia, G.: Équations elliptiques du second ordre à coefficients discontinus. Séminaire de Mathémtiques Supérieures 16, 1-77 (1966)

41. Walsh, J., Winkler, W.: Absolute Continuity and the Fine Topology. Seminar on Stochastic Processes. Progress in Probability and Statistics 1, 151-157 (1981)

42. Weis, L., Werner, D.: Reference measure and the fine topology. Preprint (1999) 